\title{
The in-situ exploration of Jupiter's radiation belts
}

\author{
Elias Roussos ${ }^{1}$ (D) . Oliver Allanson ${ }^{2}$. Nicolas André ${ }^{3}$. Bruna Bertucci ${ }^{4}$. \\ Graziella Branduardi-Raymont ${ }^{5}$. George Clark ${ }^{6}$. Konstantinos Dialynas ${ }^{7}$. \\ lannis Dandouras ${ }^{3}$. Ravindra T. Desai ${ }^{8}$. Yoshifumi Futaana ${ }^{9}$. \\ Matina Gkioulidou ${ }^{6}$. Geraint H. Jones ${ }^{5}$. Peter Kollmann ${ }^{6}$. Anna Kotova ${ }^{3}$. \\ Elena A. Kronberg ${ }^{10}$. Norbert Krupp ${ }^{11}$. Go Murakami ${ }^{12}$. Quentin Nénon ${ }^{13}$. \\ Tom Nordheim ${ }^{14}$. Benjamin Palmaerts ${ }^{15}$. Christina Plainaki ${ }^{16}$. \\ Jonathan Rae ${ }^{17}$. Daniel Santos-Costa ${ }^{18}$. Theodore Sarris ${ }^{19} \cdot$ Yuri Shprits ${ }^{20,21}$. \\ Ali Sulaiman ${ }^{22} \cdot$ Emma Woodfield $^{23} \cdot \mathrm{Xin} \mathrm{Wu}^{24} \cdot$ Zonghua $\mathrm{Yao}^{25}$
}

Received: 27 July 2020 / Accepted: 23 September 2021 / Published online: 30 October 2021

(C) The Author(s) 2021

\begin{abstract}
Jupiter has the most complex and energetic radiation belts in our Solar System and one of the most challenging space environments to measure and characterize indepth. Their hazardous environment is also a reason why so many spacecraft avoid flying directly through their most intense regions, thus explaining how Jupiter's radiation belts have kept many of their secrets so well hidden, despite having been studied for decades. In this paper we argue why these secrets are worth unveiling. Jupiter's radiation belts and the vast magnetosphere that encloses them constitute an unprecedented physical laboratory, suitable for interdisciplinary and novel scientific investigations: from studying fundamental high energy plasma physics processes which operate throughout the Universe, such as adiabatic charged particle acceleration and nonlinear wave-particle interactions, to exploiting the astrobiological consequences of energetic particle radiation. The in-situ exploration of the uninviting environment of Jupiter's radiation belts presents us with many challenges in mission design, science planning, instrumentation, and technology. We address these challenges by reviewing the different options that exist for direct and indirect observations of this unique system. We stress the need for new instruments, the value of synergistic Earth and Jupiter-based remote sensing and in-situ investigations, and the vital importance of multi-spacecraft in-situ measurements. While simultaneous, multi-point in-situ observations have long become the standard for exploring electromagnetic interactions in the inner Solar System, they have never taken place at
\end{abstract}

Elias Roussos

roussos@mps.mpg.de

Extended author information available on the last page of the article. 
Jupiter or any strongly magnetized planet besides Earth. We conclude that a dedicated multi-spacecraft mission to Jupiter is an essential and obvious way forward for exploring the planet's radiation belts. Besides guaranteeing numerous discoveries and huge leaps in our understanding of radiation belt systems, such a mission would also enable us to view Jupiter, its extended magnetosphere, moons, and rings under new light, with great benefits for space, planetary, and astrophysical sciences. For all these reasons, in-situ investigations of Jupiter's radiation belts deserve to be given a high priority in the future exploration of our Solar System. This article is based on a White Paper submitted in response to the European Space Agency's call for science themes for its Voyage 2050 programme.

Keywords Jupiter · Radiation belts · Magnetosphere · Voyage-2050 · Space missions

\section{Introduction}

\subsection{Why explore planetary radiation belts?}

Radiation belts are the regions of a magnetosphere where high energy charged particles, such as electrons, protons, and heavier ions, are trapped in large numbers. All planets in our Solar System that are sufficiently magnetized (Earth, Jupiter, Saturn, Uranus, and Neptune) host radiation belts [92, 95]. Radiation belts are not the only regions that high energy particles can be observed; they can be found throughout a planetary magnetosphere, in the heliosphere, or the astrospheres of stars, in astrophysical objects such as brown dwarfs, and in the interstellar and intergalactic medium. Many of the environments where energetic particles are found cannot be replicated in the laboratory. Even measuring particle radiation in space is not by itself sufficient to understand its origins: For instance, while we have constrained many properties of Galactic Cosmic Rays (GCRs), the highest energy particles that we can measure, their acceleration sites are inaccessible for in-situ studies.

Radiation belts offer the opportunity to perform ground truth measurements for a variety of high energy physics processes. Apart from containing the energetic particles which we can measure in-situ, they also host most mechanisms that accelerate these particles from low to high energies in a small enough region and over time scales that can be fully monitored with space missions. These processes are explored in conjunction with additional in-situ particle and fields measurements (plasma, magnetic, electric fields, electromagnetic waves) or close-proximity remote sensing observations, such as Energetic Neutral Atom (ENA) imaging [23, 93, 144, 158]. These observations, which are critical for understanding the production and dynamics of particle radiation $[177,178]$, are similarly challenging to constrain for astrophysical systems. In that sense, planetary radiation belts can be seen as laboratories for in-situ, high energy astrophysics.

The strong links between radiation belts and their host planet further advocate their exploration. Radiation belt particles are modified by the accumulated effects 
of the planetary neutral environment with which they interact: The properties of planetary exospheres, rings and moon-generated neutral torii are regularly studied through energetic particle measurements, particularly in extraterrestrial systems [38, $76,78,100,118,135]$. The reverse path, i.e. the impact of the radiation belts on different components of a planetary system, is also important: Surface sputtering or physical and chemical alteration of moon surfaces are among several fundamental consequences of such an interaction [123, 128, 131, 134].

Radiation belt measurements have been performed at all the planets hosting them. The terrestrial radiation belts, studied since the beginning of the space age, are the best understood in terms of structure, origin, and dynamical evolution [10]. However, detailed observations of other planetary radiation belts show us that not one of them can be used as a prototype for all others [139]. Generalizing our understanding of how radiation belts work requires that we realize the different ways through which particle acceleration and loss processes can be coupled in any particular magnetospheric environment.

In that respect, measurements in the radiation belts of Uranus and Neptune, sampled only once by the Voyager 2 spacecraft, should definitely be part of any future attempt to explore the two planets $[52,74]$. Saturn's radiation belts were surveyed in depth thanks to the 13-year Cassini mission at the Kronian system [139]. In comparison, Jupiter's radiation belts, while visited by numerous missions and monitored for decades through their synchrotron emission [65], still hold onto many of their secrets. No single mission, payload, or observation campaign was ever designed to capture and/or cope with their full scale, complexity, dynamics, and energetics, as argued in the two follow-up subsections.

\subsection{The uniqueness of Jupiter's radiation belts}

Jupiter's radiation belts are contained within the planet's magnetosphere, formed by a magnetic field that is 20000 times stronger than Earth's. Jupiter's fast rotation and material from Io's volcanoes that fills the system aid the magnetic field to push against the solar wind even further leading to a magnetosphere of enormous dimensions [5]. Within this giant system, the radiation belts grow into one of the most hazardous regions of our Solar System, trapping charged particles of extreme fluxes and energies that are typical for GCRs (Fig. 1). Unlike the radiation belts of Earth and Saturn that are limited in their extent [55, 144], substantial fluxes of energetic particles fill Jupiter's magnetosphere until the magnetopause [79]. Energetic electrons leaking into the solar wind are so intense that they overwhelm the $<10 \mathrm{MeV}$ GCR electrons inside $~ 10$ AU from the Sun, despite Jupiter being a point source in the vast heliosphere [137].

Jupiter's magnetic field is so strong that even ultrarelativistic, $\sim 100 \mathrm{GeV}$ protons can be trapped near the planet, over 50 times higher in energy than at Earth [15, 154]. Most importantly, observations and theory dictate that processes which may populate the radiation belts with ultrarelativistic particles do exist: Jupiter's inner radiation belts contain electrons with energies in excess of $70 \mathrm{MeV}[17,35,56]$, possibly even above $100 \mathrm{MeV}$ based on model predictions [120]. These electrons emit intense synchrotron radiation which can be detected with radio telescopes. This is 


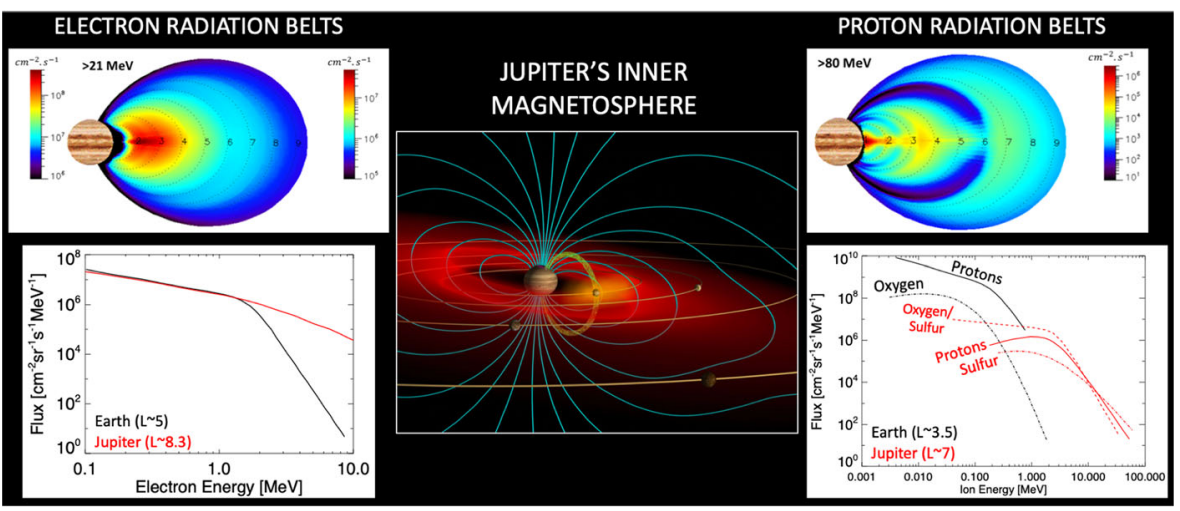

Fig. 1 Jupiter's magnetospheric region hosting the inner radiation belts (center). The moons Io, Europa, Ganymede, and Callisto are drawn, while the Io plasma torus and associated plasma disc are shown in red (Image Credit: John Spencer). Information on the inner electron and ion radiation belts are shown on each side. Color radiation belt maps are from models in [117], used with permission from Quentin Nénon. They cover the distances inward of Europa and are in part discussed in $[120,122]$. The synthetic spectra are based from data in $[92,95]$

one of Jupiter's most useful and unique qualities from an observational perspective, since a global picture of the most intense part of the belts can be seen remotely. Furthermore, data from the Juno spacecraft, currently orbiting the planet, reveal sites at locations remote from the radiation belts, where electron acceleration to $\mathrm{MeV}$ energies is regular and impulsive [19, 32, 98, 129], indicating that charged particles can gain significant energy well before reaching low jovian altitudes.

Jupiter's belts have a distinctively large variety of ions in comparable abundance to protons [2, 51] (Fig. 1, right). At other planets, ions at many $\mathrm{MeV} / \mathrm{n}$ are typically trace elements. Heavy energetic ions at Jupiter, such as oxygen and sulfur, originate primarily from its moons through volcanism or particle sputtering. At lower abundances, ions like helium, sodium, magnesium, carbon, and iron, have also been measured. Furthermore, some of the ions have a range of charge (ionization) states $[28,40,151]$. This zoo of particle species and charge states evolving across very broad energy ranges, plasma, magnetic field, and wave environments within the magnetosphere, render Jupiter into an unparalleled physics laboratory for testing theories of charged particle transport and non-adiabatic acceleration and losses [113, 120, 122].

Jupiter's magnetosphere is peppered with moons and rings which sculpt the radiation belts by absorbing energetic particles, thereby obstructing their transport and energization. Energetic particle scattering into the atmosphere through waves generated by a moon's electrodynamic interaction with the magnetosphere, as also seen at Saturn [145], can have similar effects [130, 165]. Under certain conditions, moons may instead drive charged particle acceleration, mimicking the coupling that may exist between exoplanets and their host astrosphere [157]. The presence of moons within the radiation belts is thus all the more interesting. Studying how the perturbations they generate on energetic particle distributions evolve within the magnetosphere and why moons seem to affect certain species and energies more than 
others (Fig. 1), we can gain valuable insights into the belts' complex dynamics [66, 122 , 151]. Radiation belt measurements probing physical properties of Jupiter's faint rings, that are challenging to obtain by other means, is another important application [121]. Jupiter is also considered as the closest analogue for pulsar and brown dwarf magnetospheres that could host radiation belts [70, 109, 175] (see also Section 4.4).

\subsection{Observational challenges and missing links}

From the missions that have been or are being used to gain insights into Jupiter's radiation belts (Table 1), none was designed to investigate all elements of this multi-component system in a comprehensive way. To minimize radiation exposure, spacecraft orbits or operations are often planned in ways that avoid the belts' core region where extreme levels of particle radiation are expected [136]. This region is roughly located inward of Io's orbit at $\sim 5.9 R_{J}$ and at low magnetic latitudes $\left(1 R_{J}\right.$ $=71492 \mathrm{~km}$, a jovian radius). Pioneer 10, Pioneer 11, and Voyager 1 reached deeper than Io, offering just brief snapshots of the belts from their flyby trajectories. Only few of Galileo's 34 orbits had their periapsis inward of Io's distance. Future missions JUICE and Europa Clipper will not reach deeper than Europa's orbit at $\sim 9.4 R_{J}$.

For many past spacecraft that passed through the inner radiation belts (even at distances outward of Io's orbit), their instruments suffered from saturation and/or radiation damage, rendering part of their data unusable or very challenging to calibrate $[50,171]$. Juno, the only spacecraft currently passing through the inner belts repeatedly, has instruments responding to $<1 \mathrm{MeV}$ electrons and $<10 \mathrm{MeV} / \mathrm{n}$ ions [96]. At higher energies (e.g. $>10 \mathrm{MeV}$ electrons), particles are identified through the noise they create on instruments like cameras $[12,13]$. These measurements offer unique insights into the structure of the planet's high-latitude radiation belts but are limited in energy or angular resolution. Energetic particle detectors on Galileo or earlier missions (e.g. [173]), were similarly constrained in terms of energy resolution above a few $\mathrm{MeV}$ for electrons or $\gtrsim 40 \mathrm{MeV} / \mathrm{n}$ for ions.

Energy and direction-resolved measurements are key for radiation belt studies as demonstrated by many published works for Earth and Saturn [126, 156, 164] or Jupiter (e.g. below $1 \mathrm{MeV}$ for electrons) [28, 75, 97]. Much of the electron physics at Earth and Saturn, for instance, are contained below few MeV (e.g. [9]). At Jupiter, much higher energies, which are hard to resolve, are equally important. Measurements of wave properties (e.g. chorus, Z-mode) and plasma distribution functions which impact the belts' dynamics are also limited, particularly close to the planet [107, 113], although the Juno mission is already filling some gaps from its highlatitude orbits that would also enable several deep radiation belt crossings until 2025.

Table 1 lists Earth-based observation methods that offer context information for Jupiter's radiation belts. Synchrotron emissions, currently available also from the vantage point of Juno [146], offer global views for Jupiter's electron belts, but achieve little in terms of energy resolution and provide no data on energetic ions. Monitoring of the Io plasma torus, a product of the moon's volcanic activity, offers insights about magnetospheric flow fields which control the circulation of radiation belt particles $[65,115]$, but only where the torus is present. 
Table 1 List of past, ongoing, and future missions to Jupiter's magnetosphere and radiation belts

\begin{tabular}{|c|c|c|c|c|}
\hline \multicolumn{5}{|l|}{ Space } \\
\hline Mission & Type & Time & \multicolumn{2}{|c|}{ Energetic particle measurements and constraints } \\
\hline Pioneer $10 / 11$ & Flyby & 1973-1974 & \multicolumn{2}{|c|}{ Several energetic particle detectors, saturation } \\
\hline Voyager $1 / 2$ & Flyby & 1979 & \multicolumn{2}{|c|}{ problems for protons, electrons, radiation damage } \\
\hline Ulysses & Flyby & 1992 & \multicolumn{2}{|c|}{$\begin{array}{l}\text { Several energetic particle detectors, many } \\
\text { switched off at Jupiter to avoid radiation } \\
\text { damage }\end{array}$} \\
\hline Galileo & $\begin{array}{l}\text { Orbiter, } \\
\text { Atmosphere } \\
\text { Probe }\end{array}$ & 1996-2003 & \multicolumn{2}{|c|}{$\begin{array}{l}\text { Many orbits through the equatorial belts, mostly } \\
>5 R_{J} \text { several energetic particle detectors, data } \\
\text { rate and saturation problems, radiation damage. } \\
\text { Limited data from the atmospheric probe }\end{array}$} \\
\hline Cassini & Flyby & $2000-2001$ & \multicolumn{2}{|c|}{$\begin{array}{l}\text { Distant flyby, synchrotron belts monitored by } \\
\text { radar experiment, distant ENA imaging }\end{array}$} \\
\hline New Horizons & Flyby & 2007 & \multicolumn{2}{|c|}{$\begin{array}{l}\text { Did not cross into the radiation belts' core, only } \\
\text { energetic ions below } \sim 1 \mathrm{MeV}(/ \mathrm{n})\end{array}$} \\
\hline Juno & Orbiter & 2016-2025 & \multicolumn{2}{|c|}{$\begin{array}{l}\text { Energetic particle detector, relativistic electrons } \\
\text { by monitoring noise in cameras, microwave mea- } \\
\text { surements Inner radiation belt crossings over a } \\
\text { wide latitude range. }\end{array}$} \\
\hline JUICE & Orbiter & $2031-2035$ & \multicolumn{2}{|c|}{$\begin{array}{l}\text { Mostly }>15 R_{J} \text {, energetic particle detectors }(<1 \\
\mathrm{MeV}) \text {, radiation monitor }(<40 \mathrm{MeV} \text { electrons, } \\
<250 \mathrm{MeV} \text { protons), ENA imagers }\end{array}$} \\
\hline Europa Clipper & Orbiter & 2030-2034 & \multicolumn{2}{|c|}{$\begin{array}{l}>9 R_{J} \text {, dosimeters, charge monitors for high } \\
\text { energy particles }\end{array}$} \\
\hline \multicolumn{5}{|c|}{ Other observation modes } \\
\hline \multicolumn{2}{|l|}{ Type } & \multicolumn{3}{|c|}{ Example observatories $\quad$ Characteristics and constraints } \\
\hline \multicolumn{2}{|c|}{$\begin{array}{l}\text { Synchrotron } \\
\text { Emissions, X-rays }\end{array}$} & \multicolumn{3}{|c|}{ LOFAR, GRMT, VLA $\quad<50 \mathrm{MeV}$ electrons } \\
\hline \multicolumn{2}{|c|}{ Aurora (UV, IR, X-rays) } & \multicolumn{2}{|c|}{$\begin{array}{l}\text { Hubble, XMM- } \\
\text { Newton, Chandra, } \\
\text { IRTF }\end{array}$} & $\begin{array}{l}\text { Monitoring energetic electrons } \\
(<1 \mathrm{MeV}) \text {, Heavy ions }\end{array}$ \\
\hline \multicolumn{2}{|c|}{$\begin{array}{l}\text { Io torus remote } \\
\text { sensing (UV, X-rays) }\end{array}$} & \multicolumn{2}{|l|}{ HISAKI } & $\begin{array}{l}\text { Monitoring large-scale flows, Io } \\
\text { volcanism, torus composition }\end{array}$ \\
\hline
\end{tabular}

Some constraints and challenges with respect to radiation belt measurements are indicated. Examples of remote sensing measurements with direct or indirect relevance to the jovian radiation belts (not exhaustive list) are also indicated at the lower rows. The capability for electron radiation belt monitoring with X-rays is a recent development [125]

It is clear that remote sensing observations may capture only a small fraction of the big picture. In-situ (ground-truth) observations of the radiation belts are the best way to study them, link their smallest scales to the largest, and understand their structure, origin, and dynamics. However, even with the highly anticipated scientific measurements by ongoing and future Jupiter missions in mind, a follow-up mission that aims for a dedicated and detailed in-situ investigation of the planet's radiation belts is necessary to offer closure to existing or emerging open questions. The outstanding 
science that can be performed in Jupiter's radiation belts, discussed in Section 2, justifies why such an endeavor deserves to be assigned as a high priority target in ESA's Voyage 2050 programme.

\section{Outstanding science in Jupiter's radiation belts}

Scientific investigations in Jupiter's radiation belts are well linked to three of the four overarching themes of ESA's ongoing Cosmic Vision programme, which should be relevant also for the Voyage 2050 cycle. These links are traced in Table 2 and exemplified in Sections 2.1-2.6.

\subsection{Adiabatic electron heating vs local electron radiation belt sources and losses}

State of the art and open questions Despite decades of research on how low energy electrons are accelerated to the very high energies observed in Jupiter's radiation belts, many fundamental questions remain open. Similar to Earth [62, 68, 181], two main modes of acceleration are considered: Adiabatic heating and local acceleration of electrons by nonlinear interactions with electromagnetic waves. The challenges involved in separating the two contributions arise from the fact that they overlap in time, space, and energy. In addition, the same processes may induce particle and energy losses. For example, whistler-mode chorus waves can either energize electrons through energy diffusion or scatter them into the atmosphere through pitch angle diffusion. Whether a process acts as a source or a sink depends on the background space environment which defines the energies, pitch angles, and regions that resonant interactions occur. Figure 2 summarizes our current view on how certain interactions in Jupiter's electron radiation belts (e.g. scattering) are distributed in L-shell and energy and which magnetospheric mechanism is their driving force.

Adiabatic heating, i.e. the energization of electrons through inward transport towards stronger magnetic fields, can be facilitated by at least three mechanisms:

Table 2 Jupiter radiation belt science and the links to ESA's Cosmic Vision themes

\begin{tabular}{lll}
\hline $\begin{array}{l}\text { ESA Cosmic Vison } \\
\text { 2015-2035 }\end{array}$ & $\begin{array}{l}\text { Relevant overarching scientific } \\
\text { questions for Jupiter's radiation } \\
\text { belts }\end{array}$ & $\begin{array}{l}\text { Specific science goals } \\
\text { (Subsections) }\end{array}$ \\
\hline $\begin{array}{l}\text { How does the Solar } \\
\text { System work? }\end{array}$ & $\begin{array}{l}\text { Why are Jupiter's belts so intense } \\
\text { and its magnetosphere such a pow- } \\
\text { erful accelerator? }\end{array}$ & $2.1,2.2,2.3,2.4,5$ \\
$\begin{array}{l}\text { What are the fun- } \\
\text { damental physical } \\
\text { laws of the Uni- } \\
\text { verse? }\end{array}$ & $\begin{array}{l}\text { What can we learn for high energy } \\
\text { plasma physics by studying differ- } \\
\text { ent planetary radiation belts? }\end{array}$ & $2.1,2.2,2.3,2.4,2.5$ \\
$\begin{array}{l}\text { What are the con- } \\
\text { ditions for planet } \\
\text { formation and the } \\
\text { emergence of life? }\end{array}$ & $\begin{array}{l}\text { What are the astrobiological impli- } \\
\text { cations of charged particle radiation } \\
\text { in Jupiter's mini Solar System and }\end{array}$ & $2.1,2.2,2.3,2.6$ \\
\hline
\end{tabular}






Fig. 2 A summary of processes contributing to electron transport, acceleration, and losses in Jupiter's radiation belts as a function of L-shell (magnetic equatorial distance) and energy, as we understand them today. "EMIC" refers to Electromagnetic Ion Cyclotron waves

(1) energy independent radial diffusion induced by low frequency magnetic field fluctuations possibly driven by variable thermosphere winds [22, 120], (2) interchange injections $[41,88]$, and (3) transport due to variable convective electric fields, such as the dawn-dusk electric field [115]. The latter refers to a strongly energy dependent mechanism, with high efficiency for 10-100 MeV electrons that drift slowly in magnetospheric local time ("corotation drift resonance") [141]. Existing physical radiation belt models assume an energy independent radial diffusion as available measurements cannot adequately constrain the other two processes, especially above $1 \mathrm{MeV}$. Resolving the energetic electron distribution function [164] is necessary for separating overlapping adiabatic processes with a distinct energy dependence (Fig. 2). Transient phenomena (e.g. interplanetary shocks) may also mediate adiabatic transport, but are discussed in Section 2.5.

The occurrence of resonant wave-particle interactions has been observed around Jupiter primarily by Galileo and in the extended disc of plasma outward of Io's orbit $[107,157]$. Inward of Io the space environment may also be favorable for wave-driven acceleration, but its properties are less constrained [113]. Other wave types which are not as important at Earth, such as Z-mode, may have a strong impact at the giant planets, as Saturn-based research shows [179]. The radiation belts inward of Io are a prime candidate for Z-mode acceleration of electrons [108]. These inner belts are also the only place in the Solar System where we can investigate in-situ the impact of synchrotron energy losses [36]. The production of synchrotron radiation not only affects the energy of the electrons but determines how far in latitude they can execute 
their field-aligned bounce motion, limiting in this way the wave populations they can interact with.

Key measurements and justification Figure 2 shows how any given physical process may generate a seed electron population for another to take over. Adiabatic heating may provide electrons in the appropriate energy range and L-shell where waves would accelerate them further, and vice-versa. Processes thus complement each other and are best studied in unison. In that respect, plasma moments and composition, electromagnetic wave properties (frequency, power, polarization, wave normal angle), energetic electron spectra and their spatial distribution need to be resolved. While Juno, JUICE, and Europa Clipper will bridge several existing gaps in spatial coverage [71, 90], low and mid-latitudes and a wide local time range inward of Europa would need to be surveyed. The wave frequency coverage should extend at least until up to the upper-hybrid range, such that the whistler and Z-modes are resolved at any distance, including very strong magnetic field regions near the planet. Electron measurements should be directional and energy-resolved well beyond $\sim 1$ $\mathrm{MeV}$. The signature of corotation drift resonant transport could extend up to $\sim 100$ $\mathrm{MeV}$, for instance. Observations over extended time should help define the nominal configuration of Jupiter's electron belts.

\subsection{Cosmic Ray Albedo Neutron Decay as universal proton radiation belt source}

State of the art and open questions Galactic Cosmic Rays (GCRs) with a sufficient energy to overcome the barrier of a planetary magnetic field may collide with a planet's atmosphere and rings [79]. The secondary neutrons produced by these collisions can travel away from their generation site until they $\beta$-decay to protons and electrons. If this happens in a material-free region of the magnetosphere, the decay products become trapped and add to the planet's radiation belts. This process, termed

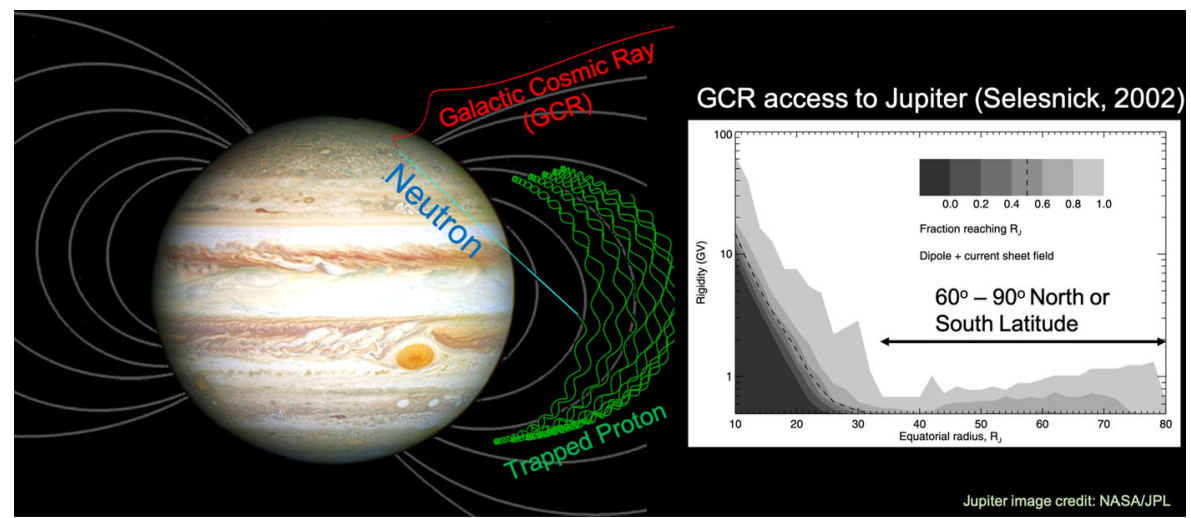

Fig. 3 The CRAND concept on Jupiter and GCR cutoff rigidities in Jupiter's magnetosphere [149]. The approximate latitude range on the planet (added by the authors) that $<1 \mathrm{GV}$ GCRs arrive $(<0.43 \mathrm{GeV}$ protons), is based on field mapping in http://www.igpp.ucla.edu/people/mvogt/mapping/ 
Cosmic Ray Albedo Neutron Decay (CRAND - Fig. 3), supplies and maintains the radiation belts of Earth and Saturn with protons of energies between several $\mathrm{MeV}$ and up to the proton trapping limit of each planet in the GeV range $[33,67,77,140$, 150]. Earth and Saturn have weaker magnetic field strengths compared to Jupiter that allow a significant part of the GCR spectrum to reach them, and material targets that can generate considerable fluxes of secondary neutrons (Earth's nitrogen/oxygen atmosphere, Saturn's icy rings). Jupiter, instead, has a hydrogenous atmosphere, tenuous rings, and a much stronger magnetic field. This different parameter regime is suitable for testing whether CRAND-driven proton radiation belts are a component of any large-scale magnetosphere or if their presence depends strongly on the unique properties of a planet and its magnetosphere.

Existing observations, with proton spectra limited below $\sim 80 \mathrm{MeV}$, do not provide any conclusive evidence: Such protons likely originate through inward adiabatic transport from a distant source [122]. Jupiter's innermost radiation belts $\left(\mathrm{r}<6 R_{J}\right)$, where very energetic protons from CRAND may get more easily trapped, are being and will be sampled extensively only by Juno. Juno, however, cannot resolve the energies of protons above $100 \mathrm{MeV}$, even if the instrument noise they create is recorded [12]. JUICE and Europa Clipper will not reach inward of Europa's orbit and will not provide constraints on CRAND, even if RADEM, the radiation monitor of JUICE, will measure protons up to $250 \mathrm{MeV}$ [133].

From a theoretical perspective, Jupiter's strong magnetic field is expected to exclude a significant part of the GCR spectrum from impacting the planet, thus restricting the production of secondary neutrons and the CRAND source rate to low values [160]. Still, that same magnetic field may form an efficient trap for CRAND protons compared to the weaker fields of Earth and Saturn. Furthermore, the GCR impact area on Jupiter is very large, while the percentage of neutrons that would decay within Jupiter's enormous magnetosphere before they escape into interplanetary space is higher than at other planets. All that could allow protons to slowly accumulate into a strong proton radiation belt despite a weak CRAND source rate. The efficiency of Jupiter's hydrogenous atmosphere in generating secondary neutrons through processes like proton-proton collisions [60] is also unknown. This efficiency has not been possible to constrain at Saturn, Uranus, and Neptune, that possess hydrogenrich atmospheres: atmospheric and ring sources of CRAND at Saturn are mixed and difficult to distinguish [34], while Voyager 2 never reached close enough to Uranus and Neptune where CRAND protons may be stably trapped (e.g. [161]).

Key measurements and justification Simulations predict that magnetospheric (nonCRAND) protons inward of Io drop significantly in intensity above 50-100 MeV and are confined to low magnetic latitudes [122]. This equatorial confinement becomes stronger inward of Thebe at $\sim 3 R_{J}$ (Fig. 1, top right). Measurements that extend the energy sampling to the $\mathrm{GeV}$ range and with a full pitch angle coverage would resolve CRAND protons directly, if present, given that CRAND is a process that can easily generate protons up to $10 \mathrm{~s}$ of $\mathrm{GeV}$. The ability to resolve the signature of CRAND would enable also a detailed monitoring of the dynamics of the energetic protons with a magnetospheric origin. 


\subsection{The enigmatic origin of the heavy and light ion radiation belts}

State of the art and open questions The jovian radiation belts contain high fluxes of sulfur and oxygen, resolved mostly up to $\sim 40 \mathrm{MeV} / \mathrm{n}$ (Section 1.2 and Fig. 1, lower right). The relative abundances of these heavy ions to protons indicate that their origin is primarily iogenic, given the lack of any other obvious sulfur source. As Io generates these species at low energies, there have to be mechanisms accelerating them to the multi-MeV/n range observed. However, the evidence on the nature of the acceleration processes are conflicting. The limited heavy ion charge (ionization) state measurements for the suprathermal energy range and challenges in resolving the ion species at many $10 \mathrm{~s}$ of $\mathrm{MeV} / \mathrm{n}$ with existing data account for that (Fig. 4A).

Singly charged energetic ions are most relevant in acceleration models which predict that charge exchange and re-ionization reactions are important [11]. These reactions transmit charged particles from the Io torus to an extended region beyond Europa's orbit, which then supplies the ion radiation belts through adiabatic transport. Radial gradients of energetic ion fluxes [30, 59] and the abundances of $>5$ $\mathrm{MeV} / \mathrm{n}$ sulfur and oxygen $[31,56]$ appear consistent with this theory. A recently discovered ion radiation belt component that includes energetic oxygen and sulfur and resides just above Jupiter's atmosphere, also requires that its ions are singly charged [75]. Theories which do not involve charge exchange and re-ionization and set no constraints on the ions' charge states, have their starting point in the enhancement of energetic ion fluxes well beyond Europa's orbit and in tail reconnection events $[80,88]$. An ion heating source at $20-25 R_{J}$, the nature of which is not understood [152], may be relevant. Indirect evidence for the presence of oxygen and sulfur with a broad distribution of charge states come e.g. from [28] through the analysis of interchange injections. [118] combine aspects of the aforementioned theories and predict that ionization states evolve with distance: Ions start as multiply charged far from Europa, gradually reducing their ionization through charge exchange as they

\section{(A)}

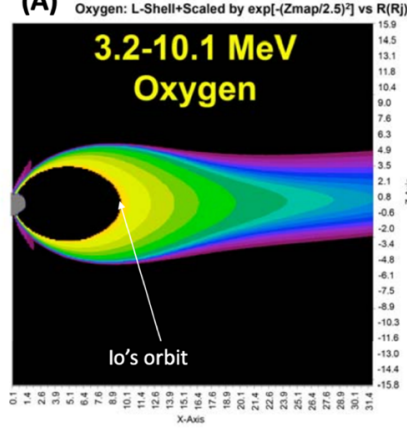

(B)

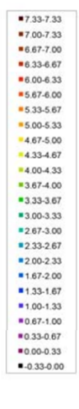

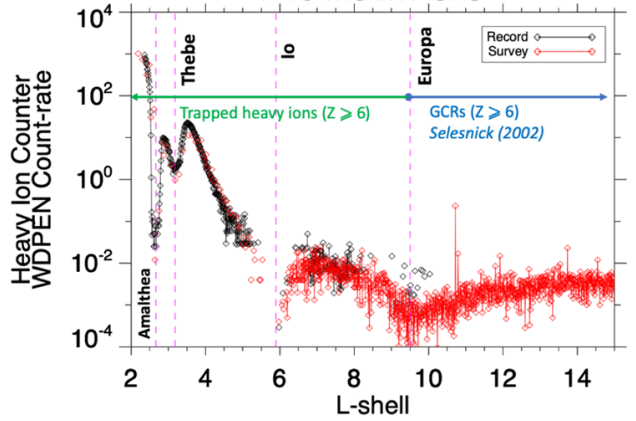

Fig. 4 (A) Integral fluxes of 3.2-10.1 MeV oxygen at Jupiter, based on an empirical radiation belt model. Figure courtesy of NASA, from [57], modified version published also in [58]. The black shaded area inward of Io's orbit is not due to missing data, but to less resolved measurements in energy and composition, shown in (B) as a mission-averaged L-shell profile of Galileo Heavy Ion Counter count-rates of $>40 \mathrm{MeV} / \mathrm{n}$ ions, partly published in [149] and [56]. Moon locations are marked, matching well the signal dropouts 
propagate through the Europa and Io torii. Still, [151], who reconstructed signatures of $\mathrm{MeV}$ oxygen and sulfur depletions seen by Galileo in the vicinity of Io, concluded that they should be fully ionized (charge states of 8 and 16, respectively). X-ray emissions from the Io torus [45] are in part consistent with fully stripped, heavy ions at $\mathrm{MeV}$ energies since electron stripping dominates over charge exchange in that energy range. Current estimates of $\mathrm{MeV}$ oxygen fluxes, however, seem too low to account for the strong X-ray signal.

At the highest observed energies, Galileo measurements inward of Io reveal a population of $>40 \mathrm{MeV} / \mathrm{n}$ and $\mathrm{Z} \geq 6$ ions which is separated from the rest of the jovian magnetosphere: A strong and persistent signal depletion is seen at Io's orbit (Fig. 4B). This implies that for $>40 \mathrm{MeV} / \mathrm{n}$ heavy ion transport across Io is difficult, if not impossible. With adiabatic transport excluded, the $>40 \mathrm{MeV} / \mathrm{n}$ species near the planet do not have an obvious source, unless they are supplied across the moon's barrier during extreme episodic events, missed by past spacecraft. If that is not the case, a local production or acceleration mechanism should be invoked. The difficulty to separate species precludes any definite conclusion.

The direct capture and trapping of heavy ions through charge stripping at the upper jovian atmosphere, as seen with Anomalous Cosmic Rays at Earth [153], may offer a pathway for heavy solar and GCR ions into the jovian system. Other energetic ion species that have been detected at Jupiter (sodium, magnesium, helium) can be used as additional tracers of the circulation and acceleration of iogenic or solar wind particles [56]. The spallation of the jovian rings as a possible source of energetic helium in the radiation belts [51] is another interesting possibility that has not been followed up.

Key measurements and justification The heavy ion radiation belts have multiple components that can be separated by both species and ionization state. Each component may be sensitive to different physical processes and its properties (energy spectra, L-shell, and pitch angle distributions) revealing for different aspects of Jupiter's radiation belts and magnetosphere. Many of the existing uncertainties arise from the fact that several ion charge state estimations were obtained indirectly. In terms of composition, Juno is filling many gaps for the $<10 \mathrm{MeV} / \mathrm{n}$ ions [63] but these need to be extended well beyond $100 \mathrm{MeV} / \mathrm{n}$, so as to understand how their acceleration evolves and what are its limits in energy. Ionization states at $<1 \mathrm{MeV}$ could still update energetic ion transport and acceleration models, in case they prove difficult to obtain for very high energies.

\subsection{High latitude charged particle acceleration as a universal radiation belt source}

State of the art and open questions Jupiter's plasma sheet is the extended equatorial region beyond Io's orbit that traps dense cold plasma and high fluxes of energetic particles mostly in the $\mathrm{keV}$ to low $\mathrm{MeV}$ range. The plasma sheet has been studied not only as a major component of the planet's magnetosphere, but also as an enormous storage ring of charged particles that supplies the radiation belts. A large body of research has been dedicated to the dynamics within Jupiter's plasma sheet (such 
as injections and ion-neutral gas interactions, Sections 2.1-2.3), because the majority of the measurements made before the Juno mission were at low latitudes. Despite that, many aspects of its energetics are still unresolved. Several Galileo-based studies reported observations of $>30 \mathrm{keV}$ electron angular distributions reminiscent of high latitude auroral processes [101, 167]. Furthermore, Galileo data show the plasma sheet to contain a low but persistent population of $>11 \mathrm{MeV}$ electrons, with no obvious origin [79]. The connection of these equatorial observations to dynamics and phenomena at high latitudes was speculated, but the lack of in-situ data from the magnetospheric high-latitude counterparts left many questions open.

Recent discoveries made by NASA's Jupiter polar orbiting spacecraft, Juno [18], have put the spotlight on auroral processes as a means to generate high-energy ions and electrons that fill Jupiter's plasma sheet. Juno has observed intense and energetic $(>1 \mathrm{MeV}$ ) electrons beaming upward from the planet [32, 98], energetic ion conics [27], and upward ion beams [99]. A remarkable discovery is the prevalence of upward electron beams. [97] have shown these to be a persistent characteristic of Jupiter's main auroral and polar cap regions. The energy flux contained in the upward beams is often greater than their precipitating counterparts and much greater than for electrons of similar energy in Jupiter's radiation belts. Similarly, in the polar cap region, upward beams of energetic electrons are always present in observations, suggesting active and persistent acceleration at altitudes below the spacecraft [97]. In addition, spectral features of Jupiter's UV aurora in the polar cap observed by Juno cannot be reconciled with the precipitating electron fluxes [44], hinting that interesting physics occur at lower altitudes that have yet to be explored. Before Juno, $>16 \mathrm{MeV}$ electrons and $<0.3 \mathrm{keV}$ protons generated at high latitudes were seen in Ulysses data [159]. Juno has detected similar features as bursts of $>10 \mathrm{MeV}$ electrons $[19,129]$. It is reasonable to hypothesize that a certain fraction of all these upward moving energetic particles populate Jupiter's plasma sheet and later end up via inward radial diffusion or injections into the radiation belts (Fig. 5). Similar hypotheses have been put forward for the $\mathrm{MeV}$ electrons produced impulsively over a large region of Saturn's high latitude magnetosphere [143], and for energetic particles from Earth's cusp [53], attesting to the possibility of high magnetospheric latitudes being an important radiation belt source, common to strongly magnetized planets with a dense atmosphere.

Key measurements and justification High-latitude auroral acceleration is a constant source of high-flux, field-aligned energetic ions and electrons. The exact details of the particles' fate are unknown and only now are theoretical ideas starting to emerge on their origin [44]. It remains unclear if, how, and what percentage of the auroral particles convert from field-aligned to trapped, populate the plasma sheet and, subsequently, the radiation belts. The equatorial counterparts of the high-latitude events may be studied partly by JUICE. Still, both JUICE and Juno offer limited information of the highest energy particles as spectral information goes to a few $\mathrm{MeV}$ and lacks energy resolution above that. Energy-resolved measurements extending to higher energies would constrain the spectral shape of the high latitude sources, determine if there is an upper limit in the acceleration for both ions and electrons, and allow us to compare if the belts' seed population shares common properties with the high latitude accelerated products. Simultaneous observations in plasma, magnetic 


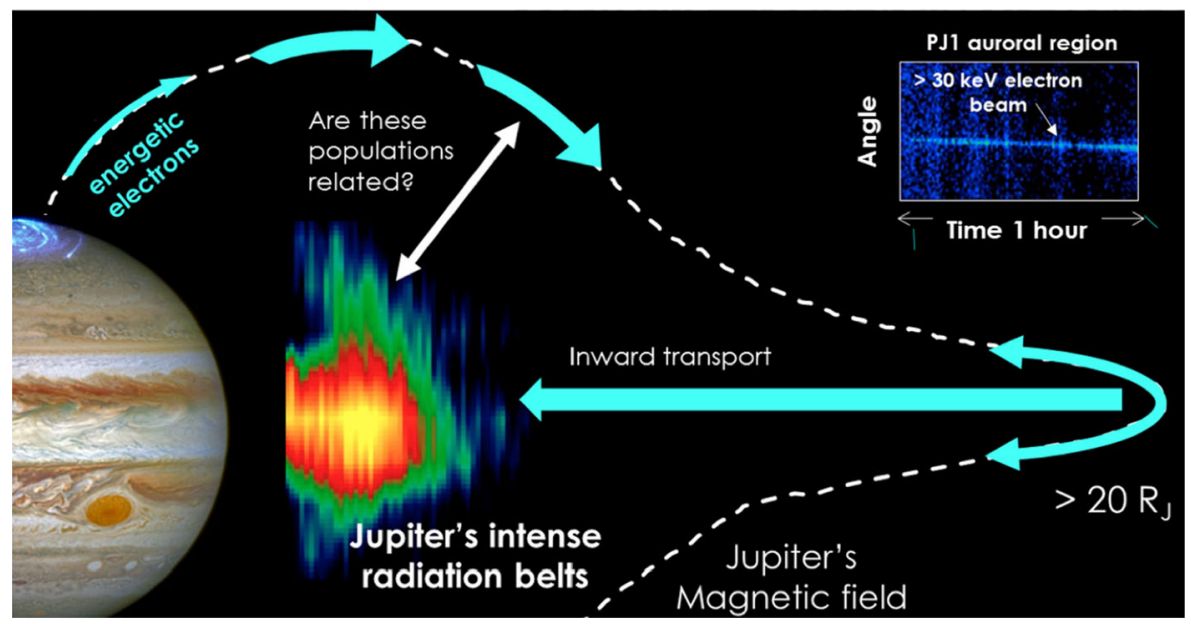

Fig. 5 The concept of high-latitude electron sources evolving into radiation belt populations. Courtesy: George Clark (JHUAPL)

field, electric field, and waves could be revealing about the origin of the acceleration mechanisms $[29,105]$.

\subsection{The space weather of Jupiter's radiation belts}

State of the art and open questions Similar to the magnetosphere that encloses them, Jupiter's radiation belts are very dynamic. This variability, i.e. the radiation belt's space weather, is important to assess for two reasons. Firstly, different physical radiation belt processes which may be difficult to distinguish in average profiles, can be resolved through their distinct variability time scales. Studies for Saturn's proton belt dynamics, for instance, have been central for understanding their CRAND-related origin $[77,140,142]$. The second reason is that there are physical interactions that are responsive to changes in a local radiation belt environment, such as surface sputtering creating transient satellite exospheres [110].

Most of our information for time variations in Jupiter's radiation belts comes from monitoring the synchrotron emissions by their MeV electrons. These reveal changes at different time scales, ranging from days to years. The longest-term changes seem to have a two-year lagged response to the solar wind [35, 37, 54, 147]. It has been argued that this time lag is explained by slow radial diffusion of electrons which is mediated through a solar-wind modulated dawn-dusk electric field that has been observed near Io's orbit $[65,115]$. If this model is correct, radiation belt transients should evolve the fastest for 10-100 MeV electrons (Fig. 2, "corotation resonance"). Such rapid enhancements in $>11 \mathrm{MeV}$ electron data were observed in Galileo data [141], but the lack of energy resolution for these measurements, prevents any definite conclusion. How the solar wind transmits changes in the dawn-dusk electric field at Io is also unknown, in part because instantaneous solar wind parameters upstream of Jupiter come from models propagating measurements obtained at 1 AU to the planet's 
heliocentric distance at 5.2 AU. Furthermore, the dawn-dusk electric field has never been detected in-situ, in plasma flows, or electric field measurements [7]. An in-situ detection could verify if this convective field extends inward or outward of Io's orbit and indicate the spatial scales over which it exerts control on the radiation belts.

Regular, short-term variations in the synchrotron belts ( $\sim$ days) have been observed in response to solar EUV flux changes, supporting a theoretical link between radial diffusion of electrons from changing winds in the planet's thermosphere [22, 73, 168]. Measurement of electron spectra following EUV changes, for exploiting this link in-situ, are missing. Such measurements could peer into the physics of thermosphere-driven diffusion: Is this process energy dependent and how does it scale with L-shell? At Saturn, for instance, where there is evidence that this form of diffusion acts on its radiation belts, in-situ data show that this scaling is much steeper than theory predicts $[77,138]$. The least explained variations are those on time scales of weeks, which show no obvious correlation to either EUV or dawn-dusk electric field changes [72].

For the ion radiation belts of Jupiter that cannot be resolved through synchrotron emissions, variations are poorly explored. Unlike Saturn, whose moons restrict $\mathrm{MeV}$ ion transport, at Jupiter this does not occur, at least below the $40 \mathrm{MeV} / \mathrm{n}$ range ([122] and Section 2.3). Communication between energetic ion populations across the moon orbits should be possible, such that variations in the middle magnetosphere may be transmitted to the innermost belts. For all species, a several day recurrence rate of tail reconnection events, which likely enhances the seed radiation belt population [84, 88], may be important. Juno findings of high latitude particle acceleration sites may also be significant, if these indeed regulate the belt's seed population (Section 2.4).

For certain ion species, the lack of variations may be revealing. For instance, since CRAND (Section 2.2) is a quasi-stable source, seeking for a steady proton signal over multiple orbits would allow to distinguish it from other, more variable sources of protons. If the population of energetic heavy ions $(>40 \mathrm{MeV} / \mathrm{n})$ is stable over even long timescales, as hinted in data shown in Fig. 4B, its source process would need to be equally invariant with time.

Key measurements and justification Any of the aforementioned open questions would benefit from the in-situ measurements identified for all science themes discussed in Sections 2.1-2.4, collected over extended time periods to enable variability studies. Different time scales can be probed by regulating the re-sampling rate of the radiation belts, as done e.g. for Saturn with Cassini $[141,144]$. Simultaneous solar wind measurements are important to obtain close to Jupiter. Capturing the global dynamics of the belts' seed population (beyond Europa's orbit), could be achieved through ENA and/or X-ray imaging. Remote sensing of the Io plasma torus, the aurora, and the synchrotron emissions of the electron belts would provide excellent context (Fig. 6).

\subsection{Radiation processing of moon surface material and astrobiological implications}

State of the art and open questions Excluding Io, the surface of which is regularly altered by its ongoing volcanic activity, all other moons of Jupiter that orbit within the 
magnetosphere and the radiation belts are exposed to a variety of external weathering processes that modify their physical and chemical properties. Irradiation by low and high energy charged particles is one of these processes. The radiation belt particles, which are also the most surface penetrating, can reach between several $\mathrm{cm}$ to several $m$ in depth, either directly or due to the Bremsstrahlung they emit as they are decelerated in ice $[123,124]$. Such interactions can transform the top layers of the surface by altering its crystallinity, its thermal inertia, or even by creating new molecules ([128] and references therein). These modifications should be considered when interpreting remote sensing observations of the moons (e.g. reflectance spectra), when surface samples are retrieved directly through a lander, or indirectly, by measuring sputtered products and micrometeoroid ejected dust grains from the surface, as it will be done with JUICE and Europa Clipper.

For Europa, where future landers could seek biosignatures in material that may have upwelled from its subsurface ocean, the study of radiation-induced surface modifications has an added value. Particle radiation can impact Europa in different ways, for example by generating the already detected $\mathrm{H}_{2} \mathrm{O}_{2}$ on its surface [24]. This oxidant may provide a source of chemical energy to sustain a biosphere in Europa's ocean $[26,169]$. On the other hand, it is estimated that aminoacids, the simplest compounds to be sought as potential biosignatures on the surface, can be destroyed by particle radiation from Jupiter down to a depth of about $10 \mathrm{~cm}$ in Europa's equatorial region (Fig. 7) [123].

Quantifying any of the mechanisms discussed above depends significantly on the input energetic particle spectra to which a surface we assume is exposed. Particularly

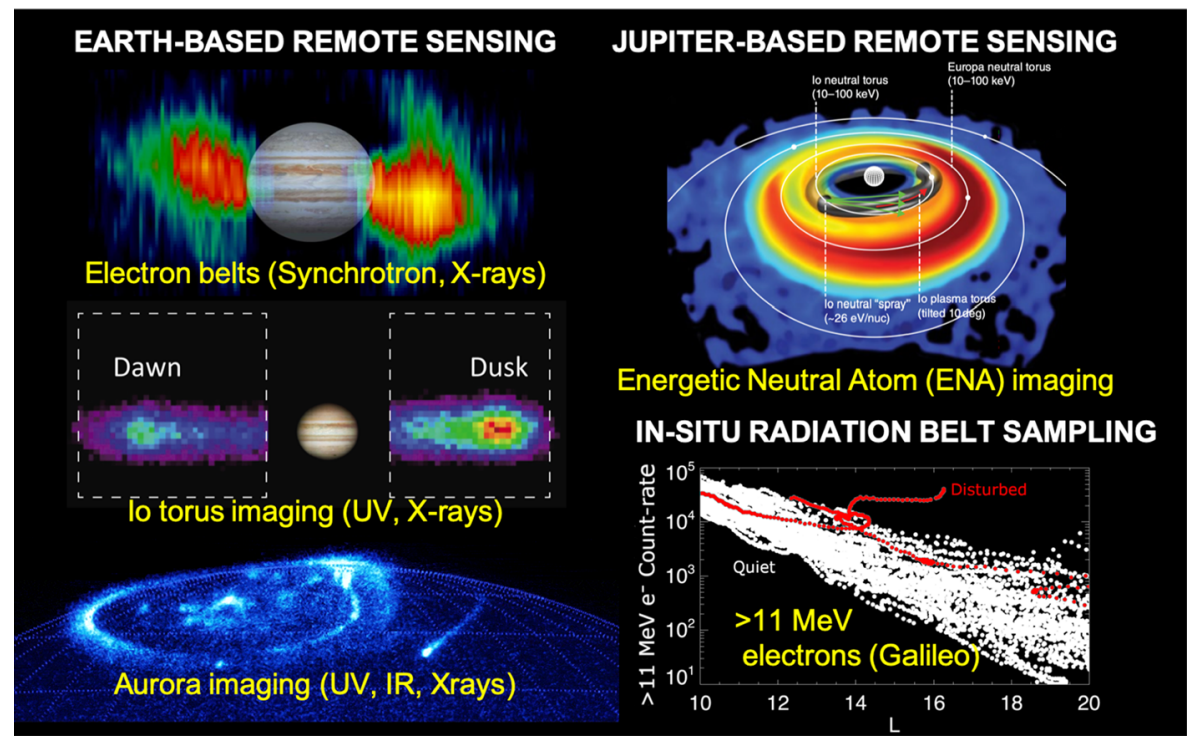

Fig. 6 The different options for space weather monitoring of Jupiter's radiation belts. Image/plot credits: synchrotron belts (NASA/JPL), torus imaging (from [115], used with permission), aurora image (NASA/STScI), ENA image simulation (from [20], used with permission), in-situ data plot based on [141] 

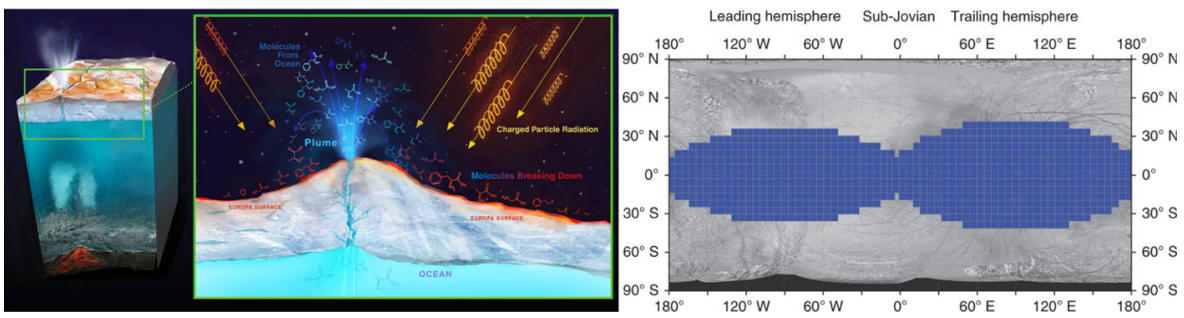

Fig. 7 (Left) Europa's surface is continuously bombarded by charged particle radiation (Image credit: NASA/JPL). The blue-shaded area (right) shows the current best estimate on where Europa's surface is radiation-processed down to $\sim 10 \mathrm{~cm}$ (from [123], used with permission)

for $>1 \mathrm{MeV}$ electrons and $>10 \mathrm{MeV}(/ \mathrm{n})$ protons and ions, energy spectra are not as well constrained as for lower energies. Many of the relevant studies rely on arbitrary extrapolations to energies of $100 \mathrm{~s} \mathrm{MeV}(/ \mathrm{n})$ or more to predict the most penetrating particles' effects. For even higher energies, it is assumed that the particle spectrum is that of GCRs that can reach into a moon's orbit [124], resulting in rather low input fluxes for most moons: in Europa's case, $<13$ GeV GCRs cannot reach its orbit [124, 149]. However, if processes like CRAND provide even a small input to Jupiter's radiation belts (Section 2.2), fluxes of relativistic protons can be significantly elevated from their presumed "empty" GCR levels. Proton trapping at Europa is limited at $\sim 2.5 \mathrm{GeV}$ and exceeds $100 \mathrm{GeV}$ near the planet [15]. CRAND protons would populate the spectral range below the GCR cutoff energy, up to the trapping limit.

Key measurements and justification An improved description of the energetic particle spectra would transform our understanding of how Jupiter's radiation belt environment affects the surfaces of its moons. All effects discussed here develop on timescales of thousands to millions of years, such that long-term average energetic particle spectra should be sufficient to estimate the energy flux and its long-term impact on the moons' visible surface and subsurface. The requirements for the energy range that the measurements should cover are similar to those discussed in Sections 2.1-2.5. Understanding if certain physical processes, like CRAND, contribute to the radiation belt content, would allow to physically extrapolate measurements to even higher energies than it would be possible to detect.

\section{Space missions to Jupiter's radiation belts}

Here we outline considerations for space missions that could explore Jupiter's radiation belts and we identify enabling technologies. Several past studies by ESA were done in the context of the JUICE mission, including its previous variants, Laplace and EJSM. Even earlier studies of relevance are those of the Jupiter Radiation Study (JURA) and a series of investigations for the exploration the jovian system by [4]. The latter were subdivided into three mission studies, the Jovian Minisat Explorer (JME), the Jupiter Entry Probe (JEP), and the Jovian System Explorer (JSE), focusing on Europa, the jovian atmosphere, and the magnetosphere, respectively. The 
JUpiter MagnetosPheric boundary ExploreR (JUMPER) study is likewise relevant [43]. Appropriate points from those reports are reiterated here. Besides that, the mission scenarios discussed in Section 3.3, which are only introduced as initial concepts, deserve dedicated studies on both mission and subsystem levels.

\subsection{Scientific design drivers}

Spatial coverage Studying the radiation belts requires to monitor them as part of the jovian magnetosphere. Spatial coverage should be maximized in terms of distance (from the planet to the magnetopause), latitude, and local time, similar to what Cassini achieved at Saturn or Juno is currently doing at Jupiter. Should that not be possible, the least explored regions should be prioritized, i.e. the radiation belts' core between the planet and Io at low and mid-latitudes. In terms of local time, strong asymmetries are expected between the dawn and dusk magnetospheric sectors based on remote sensing observations (e.g. [115]). Day-night asymmetries cannot be resolved by Earth-based observations, but may be constrained by Juno (e.g. [146]), and based on future findings, priorities for local time coverage should be modified accordingly.

Temporal coverage The radiation belts evolve on a variety of timescales, the longest ones in the order of a few years. The minimum lifetime of a single mission to Jupiter's belts should be about 2 years. Resolving shorter time scales (weeks/months) could be achieved through radiation belt crossings with a similar frequency. For even shorter time scales and for separating temporal from spatial variations, multi-point, in-situ measurements in the magnetosphere and the synergy between remote sensing and in-situ observations are key. Remote sensing may be offered through Earth-based observatories (e.g. synchrotron observations - Table 1, Fig. 6) or from Jupiter, at a wide range of wavelengths as well as in ENAs.

Instrumentation While the radiation belts are described by their energetic particle distributions, their origin, structure, and dynamics are best understood through complementary investigations of their space environment in plasma, magnetic field, electric field, and waves at a wide frequency range (Sections 1, 2). Table 3 marks which type of instruments are relevant for different investigations. The properties for the particles and fields suite of Juno, JUICE, Europa Clipper, and the Van Allen Probes $[18,61,162]$, offer excellent analogues for the requirements of similar detectors in a mission to the radiation belts. An added measurement capability should be that of energetic ion charge states, as done with the CHEMS instrument on Cassini [81]. Among the instrument types listed in Table 3, the most novel would be those for detecting (ultra)relativistic particles. Extending energy and direction resolved measurement capabilities to $\sim 100 \mathrm{MeV}$ electrons and $\sim 1 \mathrm{GeV}(/ \mathrm{n})$ ions, in order to comply with expectations for the energies of trapped populations in Jupiter's radiation belts, has never been attempted in a planet other than Earth or the solar wind around $1 \mathrm{AU}$, where large detectors, mostly aiming at low flux GCRs, have been or are being operated (e.g. [132]). Measurements of particle distributions for all energies requires $4 \pi$ sky coverage for ions or full pitch angle coverage for electrons, such 


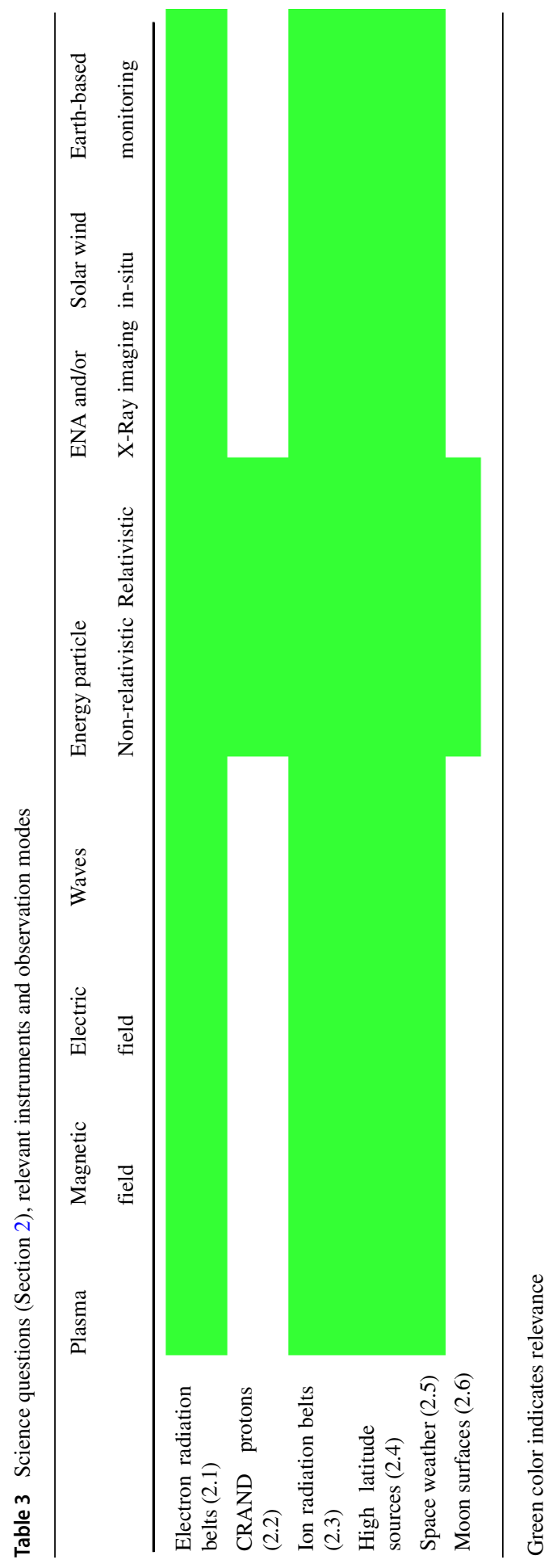


that a spinning spacecraft is preferred over a three-axis stabilized one. A spinning spacecraft may have additional advantages for instrument design (Section 3.4).

\subsection{Technical design drivers}

While there are numerous technical design drivers, below we focus on those with unique aspects for a mission to Jupiter's radiation belts (mass, radiation, communications, planetary protection). Discussion about parameters such as power, thermal constraints, spacecraft autonomy are addressed in past Jupiter mission studies.

Mass The payload masses for Juno, JUICE, and Europa Clipper are $\sim 170, \sim 220$ and $\sim 350 \mathrm{~kg}$ respectively $[18,39,46,116]$, with typically less than $40 \%$ allocated for in-situ particles and fields instrumentation and ENA imagers. While that appears to imply significant extra mass for accommodating improved and new instruments for a dedicated space physics payload, the available margin may be significantly smaller: The required, multiple crossings through the radiation belts translate to heavier radiation shielding. The possible use of instruments with strong magnets may also require additional material for magnetic shielding (Section 3.4). The need for solar wind monitoring and multi-point in-situ measurements implies mission designs with at least two orbiting spacecraft, setting tight mass constraints for the case these have to be accommodated in a single launcher. From earlier mission studies [4, 43], the minimum mass of a second, small orbiter would be in the range of $100 \mathrm{~kg}$. Payload distribution among the different spacecraft, orbit, instrument, and shielding design are parameters that need to be iterated for maximizing payload mass and optimizing science return.

Radiation For each of its few orbits through the inner radiation belts the Galileo spacecraft was accumulating 30-40 krad per crossing behind an equivalent shielding of $2.2 \mathrm{~g} / \mathrm{cm}^{2}$ aluminum [3,50], in agreement with estimates from radiation belt models [136]. At the center of the radiation belts core, the same models estimate dose rates that can be as high as 200 or $100 \mathrm{krad} /$ day behind $2.2 \mathrm{or} 5.0 \mathrm{~g} / \mathrm{cm}^{2}$ aluminum, respectively. This means that within several orbits, a spacecraft crossing through the innermost radiation belts core with a shielding below $5.0 \mathrm{~g} / \mathrm{cm}^{2}$ would accumulate an equivalent dose as Galileo during its 6.5 years and 34 orbits mission. Clearly, much heavier passive shielding than $5.0 \mathrm{~g} / \mathrm{cm}^{2}$ would be required. On the other hand, given the unavoidable constraints in mass, radiation mitigation strategies should also iterate orbit design, spacecraft and instrument shielding distribution, instrument and subsystem accommodation and aim for the development and use of radiation-hard electronics, to minimize radiation dose accumulation per orbit, to extend subsystem tolerance and overall maximize mission lifetime and the number of radiation belt crossings. Heritage from past missions and particularly the measurements and operations of the Juno spacecraft, that is repeatedly crossing through the inner radiation belts, would be valuable for defining radiation mitigation strategies.

Communications In the case of a multi-spacecraft mission the options for autonomous (Earth-direct) vs intra-spacecraft (relay) communications should be 
examined as they impact orbit and spacecraft design. Direct-to-Earth communications likely allow for higher flexibility and autonomy to each spacecraft for science operations. A relay offers the option of longer storage and downlink periods of large volume of high-quality data [4].

Planetary protection Europa's surface or immediate subsurface (see Section 2.6) may be hosting biosignatures associated to the subsurface ocean below its crust, and thus planetary protection considerations are relevant, especially for a mission that may cross the moon's orbit and a spacecraft that is subject to risks imposed by very high radiation exposure. In terms of orbit, the design should minimize the possibility of an accidental impact with the moon. In the study for the Jovian Minisat Explorer it was considered that in-flight decontamination by the extreme radiation in Jupiter's belts may be an option that could be explored.

\subsection{Space mission considerations}

Monitoring of the radiation belts through one or more orbiters is necessary for their comprehensive exploration. Several mission concepts are outlined, each one for different mission cost levels (L- or M-class). A third flyby or short-duration, low altitude orbiter mission option is described as a target-of-opportunity or a pre-cursor to options (1) and (2) (Fig. 8). Any of the three scenarios would benefit from coordinated Earth-based monitoring of the jovian system (Table 1). For any of the mission concepts, international collaboration may reduce ESA's contribution and depending on the level of involvement the cost-category of a mission scenario may change. All concepts are principally feasible on solar power, although other options involving space nuclear power [166] may potentially allow for extra flexibility in mission design.



Fig. 8 Simplified sketch for orbiter mission concepts "1" and "2" (top). Option "3" (flyby or short-lived, low altitude orbiter) is shown at the bottom panel. Distances shown are not to scale 
Option 1: Radiation belt orbiter and Solar wind/magnetosphere monitor Covering all science goals identified in Section 2 requires synergistic investigations by a minimum of two orbiters. For the minimal configuration we consider that a primary spacecraft performing regular radiation belt crossings carries the in-situ particles and fields instrumentation suite, while a smaller spacecraft monitors the upstream solar wind and the magnetosphere globally.

The primary spacecraft's orbit should be highly elliptical, similar to Juno's ( $\sim 53$ days period), to minimize crossing durations through the belts and exposure to radiation, but more equatorial so as to sample the radiation belts at their core. As both Juno and Cassini investigations already demonstrated, belt crossings lasting several hours each are more than sufficient for collecting excellent quality observations and for resolving radiation belt structuring down to $\sim 10^{-2}$ planetary radii thanks to the hightime resolution sampling that is possible for all particles and fields instruments [75, 140]. As the mission evolves, the eccentricity and the period of the orbits could be reduced so as to achieve more frequent belt crossings and capture shorter variability time scales. Excursions in local time and latitude could be performed as the apojove reduces, e.g. through Callisto flybys, like it will be done for JUICE, or with spacecraft resources, if possible. Latitudinal excursions could regulate the rate of ionizing dose accumulation as the belt crossings are becoming more and more frequent.

The secondary spacecraft, carrying a lightweight in-situ particles and fields suite and remote sensing instruments (e.g. [43]), should be placed in an orbit extending upstream of Jupiter's bow-shock $\left(\sim 100 R_{J}\right)$ from where solar wind monitoring is possible for extended periods. Its perijove could be outside Callisto's orbit to limit radiation exposure. Closer to the magnetosphere, ENA and X-Ray imaging becomes possible, along with simultaneous two-point measurements with the primary orbiter. If only one imager can be accommodated, an ENA camera would be preferred if all the other remote sensing methods that can be executed from Earth (Table 1) are available. X-ray imagers offer otherwise excellent remote sensing options, as they may probe simultaneously the electron belts, the aurora, and the Io torus [21, 42, 45, 124].

Option 2: Radiation belt orbiter The M-class variant is similar to the L-class but does not include the solar wind/magnetosphere monitoring spacecraft. Most science goals that depend on solar wind monitoring, are affected. Losses can be partly mitigated by propagating solar wind parameters from $1 \mathrm{AU}$. Close-proximity ENA or X-Ray imaging and two point in-situ measurements in the magnetosphere may not be replicated, unless any of these imagers can be accommodated on the primary spacecraft. The advantages of a single orbiter are that mission design and operations would be simpler, while significant mass allocation could become available for instrument design, radiation shielding, or other critical mission subsystems. This variant retains a great discovery potential, given the existing gaps in spatial and temporal coverage of Jupiter's radiation belts as well as missing measurements (Section 1.3).

Option 3: Flyby or short-duration orbiter Due to its strong gravity, Jupiter is regularly used as a swing-by target for adjusting a spacecraft's trajectory towards its Solar System destination. Many mission concepts target the Jupiter system itself. A Cubesat 
or Smallsat-sized probe riding along with the main spacecraft could be released for a flyby through the belts' core. For such a small resource mission, observations should prioritize measuring the least defined parameters, i.e. suprathermal ion charge states, electrons up to $\sim 100 \mathrm{MeV}$, protons up to $\sim 1 \mathrm{GeV}$ and ion composition at least up to $100 \mathrm{MeV} / \mathrm{n}$. Observations from such a flyby mission could provide key input for science goals 2.2, 2.3, and 2.6, further constraints to all others and refine the requirements for Options 1 and 2. Should resources allow, a small, short-lived orbiter with frequent crossings through the belts' core can be considered instead. Such low resource missions can be successful: Data from the Colorado Student Space Weather Experiment CubeSat in Earth's radiation belts, for instance, led to the discovery of the decades-sought, trapped CRAND electrons [85, 86].

\subsection{Enabling technologies}

Miniaturization of relativistic charged particle detectors For the high energies considered in Jupiter's radiation belts $(\sim 100 \mathrm{MeV}$ electrons, $\sim \mathrm{GeV} /(\mathrm{n})$ ions), particles are both penetrating and fast. Methods measuring time-of-flight or energy losses on silicon detector stacks either don't work or give poor constraints to a particle's energy, e.g. above 100-200 MeV for protons. A most efficient method to resolve energy, species, and ionization state of such particles is magnetic spectrometry, used until now in planetary radiation belts but for electrons in the $\mathrm{keV}$ to low $\mathrm{MeV}$ range (e.g. $[16,81])$. Existing detectors that use it for higher energies are very power consuming and heavy (many 10s of kg or more) partly due to the application of large permanent magnets [132] and partly because they are designed to detect low flux, ultra-high energy GCRs. A step towards miniaturizing such instruments for planetary applications is the MiniPAN (Mini-Penetrating Particle Analyzer) detector [180], a modular magnetic spectrometer (mass $<10 \mathrm{~kg}$, power $<20 \mathrm{~W}$ ) that may perform ion composition and electron spectrometry measurements up to several $\mathrm{GeV}(/ \mathrm{n})$ (Fig. 9). A demonstrator was approved for development through a European Union Future and Emerging Technologies program, but a Jupiter-specific design would require further studies.

The energy limit to which magnetic spectrometry can be applied for resolving heavy ion ionization states remains to be investigated. Non-magnetic spectrometers for ultrarelativistic electrons developed in Europe, such as HEPD that is operating on the China Seismo-Electromagnetic Satellite [1], use silicon detector stacks and calorimeters. The large mass of all aforementioned detectors may be reduced by adjusting their geometry factor to the orders of magnitude stronger $\mathrm{MeV}$ electron and ion fluxes at Jupiter compared to that of GCRs (Fig. 1). Additionally, such instruments reconstruct the 3D trajectory of the measured particles by tracking them on large, successive detector planes of silicon strip detectors. With an instrument on a spinning spacecraft (Section 3.1) tracking becomes two-dimensional, which may also allow to reduce its total mass as slit-shaped detectors can be accommodated in smaller volume.

Electromagnetic cleanliness For magnetic spectrometry to capture the energies of particles in Jupiter's radiation belts, permanent magnets should generate static fields 

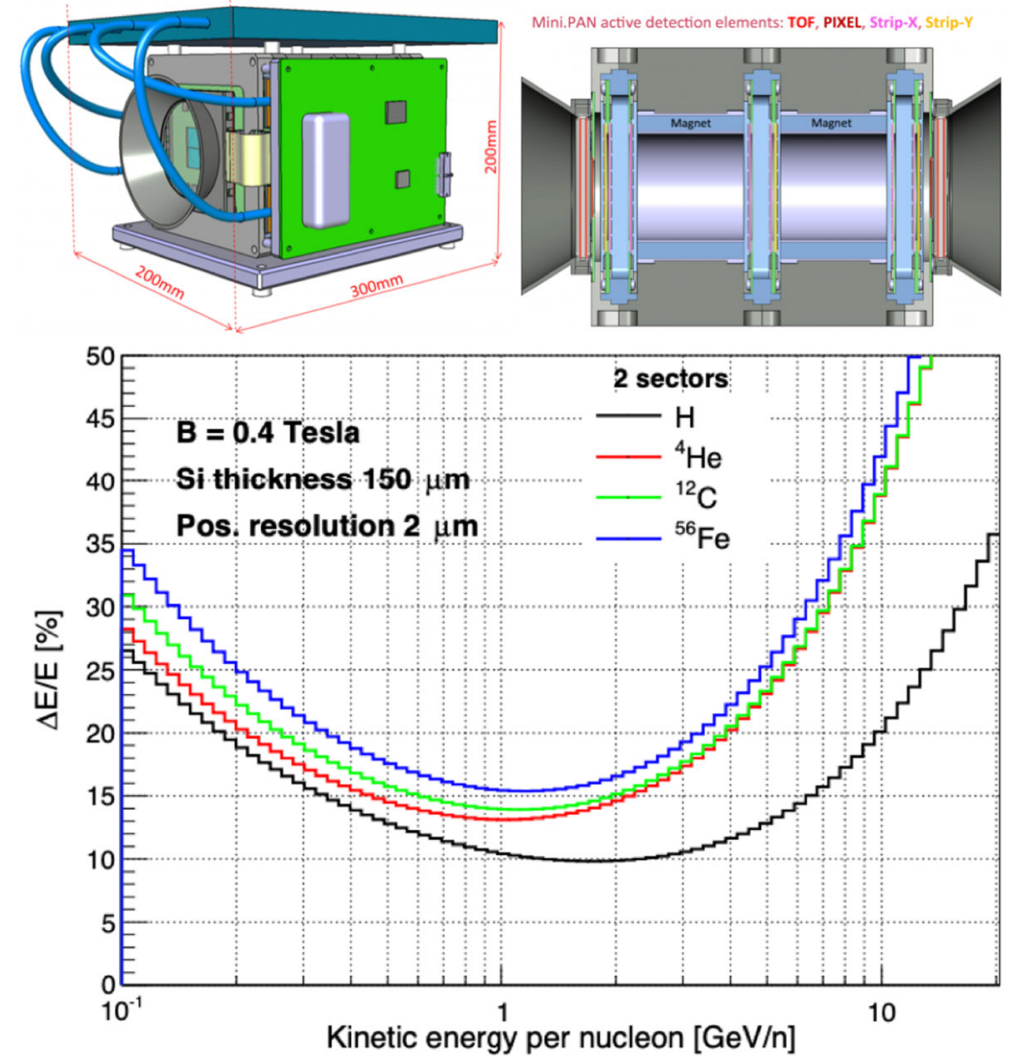

Fig. 9 (Top panels) Simplified schematic of a double-sided MiniPAN instrument concept. (Bottom) The estimated energy resolution as a function of ion kinetic energy, for different ion species. Images are from https://www.unige.ch/dpnc/index.php?cID=947 and more information is available in [180]

that may have to be several times stronger than what has been used e.g. on the LEMMS instruments of Cassini and Galileo ( 0.06-0.08 T) [81, 173] or the MagEIS instrument of the Van Allen probes $(\sim 0.15 \mathrm{~T})[16]$ or be considerably larger in size. Strong stray fields from permanent magnets can interfere with plasma detectors or magnetometers that are essential for any radiation belt focused mission. The trade-off between the maximum energy resolved (determined by the magnet strength and size), the strength of the stray fields and instrument mass should be considered. Instrument accommodation and design, e.g. choice of materials for the permanent magnet and for magnetic shielding, play a role.

Radiation hard electronics and detector performance The JUICE mission is designed such that the Total Ionizing Dose (TID) experienced by its most sensitive electronics contained in heavily shielded vaults would not exceed $50 \mathrm{krad}$ by the end of the mission [46]. This tolerance level should be increased for a dedicated radiation belt mission. In addition, developing efficient active shielding systems for particle 
detectors (e.g. anticoincidence or positive coincidence) that would reject counts from high fluxes of penetrating $\mathrm{MeV}$ electrons and protons in Jupiter's belts, should be investigated. Low energy electron detectors $(<10 \mathrm{keV})$ are the most challenging for using anticoincidence [e.g. [102]], as techniques like Time-of-Flight, cannot be applied, and other coincidence schemes should be explored. The use of Faraday Cups as on Europa Clipper, which are less sensitive to high energy radiation, offer a solution for the measurement of low energy particles [172]. Both the high-resistant electronics and active shielding techniques, would reduce the requirements for passive spacecraft shielding as the way to achieve a good signal to noise ratio, allowing to allocate mass in other subsystems.

Energetic Neutral Atom imaging ( $>1 \mathrm{keV}$ ) ENA imaging is considered in the payload concept described in Section 3.1. JUICE will be first mission to perform a dedicated study at Jupiter in low and high energy ENAs [61]. Still, detectors for $>1 \mathrm{keV}$ ENAs (INCA on Cassini, JENI on JUICE) [111], have been or are being developed only outside Europe. Developing high energy ENA capabilities would enable more flexibility in mission planning and open new roads for scientific applications in Earth's magnetosphere, comets, and the heliosphere where ENA imaging is applicable [20, 42, 103].

\section{Jupiter radiation belt science in context}

\subsection{Jupiter exploration}

Following the exciting discoveries by the Galileo mission at Jupiter, JUICE and Europa Clipper were designed in response. Besides exploring Europa, Ganymede, and Callisto in detail, these missions will carry instruments that offer an interdisciplinary view of the jovian system, including its magnetosphere and the outer radiation belt regions. Similarly, new discoveries by Juno, which has been extended through September 2025, are revealing the complexity of the jovian magnetosphere and its energetic particle environment, already generating many new questions (Section 2.4). These missions, along with campaigns by Earth-based observatories, would shape our views about Jupiter until about 2035 (Fig. 10). Beyond 2035, a mission that may explore Jupiter's magnetospheric environment has been announced by China, with arrival probably after 2036, but with no further details known [25]. A space mission, picking up from where Juno, JUICE and Europa Clipper take our understanding, will continue the exploration of the giant planet, which has been regular since Pioneer 10 and 11 (Table 1). Jupiter exploration has also benefited from the synergy of space missions and Earth-based observatories. The prospects for the development of new observatories after 2035 are still unclear.

\subsection{Multi-spacecraft investigations of planetary magnetospheres}

Over the last two decades, terrestrial magnetospheric science has benefited greatly from space missions utilizing several spacecraft for simultaneous, multi-point 


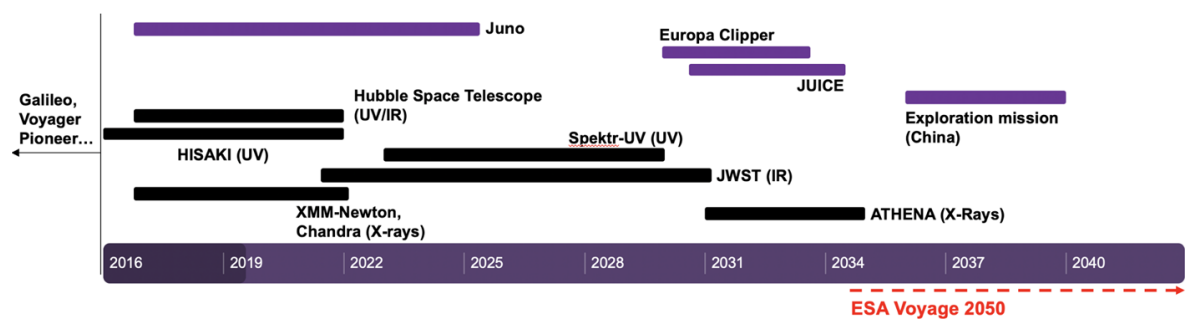

Fig. 10 The timeline of missions relevant to Jupiter's magnetosphere and radiation belts (magenta: space missions; black: Earth-based observatories that can target Jupiter). Ground-based, synchrotron radiation belt or IR, jovian aurora observations are assumed to be available throughout this timeline. The timeline for China's exploration mission is tentative. For other future missions, timelines indicate the anticipated period of science operations. As of January 2021, Juno has been extended until September 2025

measurements of the geospace, in addition to the regular monitoring of the upstream solar wind and of the various geomagnetic indices which has been going on for even longer. Missions like Cluster, Double Star, THEMIS, Magnetospheric Multi-Scale (MMS), and the Van Allen Probes have drastically changed the way we understand Earth's radiation belts, as part of the terrestrial magnetosphere (e.g. [47]). The findings from the Van Allen Probes, in particular, have led to many breakthrough discoveries and have evolved the state of the art of radiation belts' research to a new level $[8,126,156,163]$. The remarkable increase of refereed publications on the subject after 2000 (Fig. 11) and the launch of follow-up missions like Arase, the China Seismo-Electromagnetic Satellite, Lomonosov, and others [1, 112, 155], are representative of the aforementioned, rapid progress and increased interest in the field (see also Appendix 1). Multi-point measurements of space plasma interactions in our Solar System have become a norm: The two ARTEMIS spacecraft investigate the lunar-solar wind interaction since 2010 [64], while BepiColombo will do the same at Mercury [14]. The recently funded ESCAPADE mission will be the first dedicated two-orbiter mission to study the Mars-solar wind interaction [87]. Similarly, ESA's Comet Interceptor will perform two-point measurements of a comet's plasma environment [69]. As it stands now, no coordinated multi-spacecraft mission is planned for any outer planet magnetosphere, but it is the essential and obvious way forward. A mission to explore Jupiter's radiation belts (Section 3.2) would have an enormous potential for discoveries, not only because it could be the first to investigate Jupiter's magnetosphere with multiple spacecraft, but also because it would focus on one of its least sampled components.

\subsection{Comparative studies}

Jupiter radiation belt science is well aligned with the continuous investigations of Earth's radiation belts by numerous ongoing missions, and the fact that many White Papers in response to ESA's Voyage 2050 call focus on charged particle acceleration and energy transfer between plasmas and fields. Thanks to the extensive exploration of Saturn's radiation belts by Cassini and the continuously growing set of measurements by Juno at Jupiter, a large volume of high-quality observations is being 


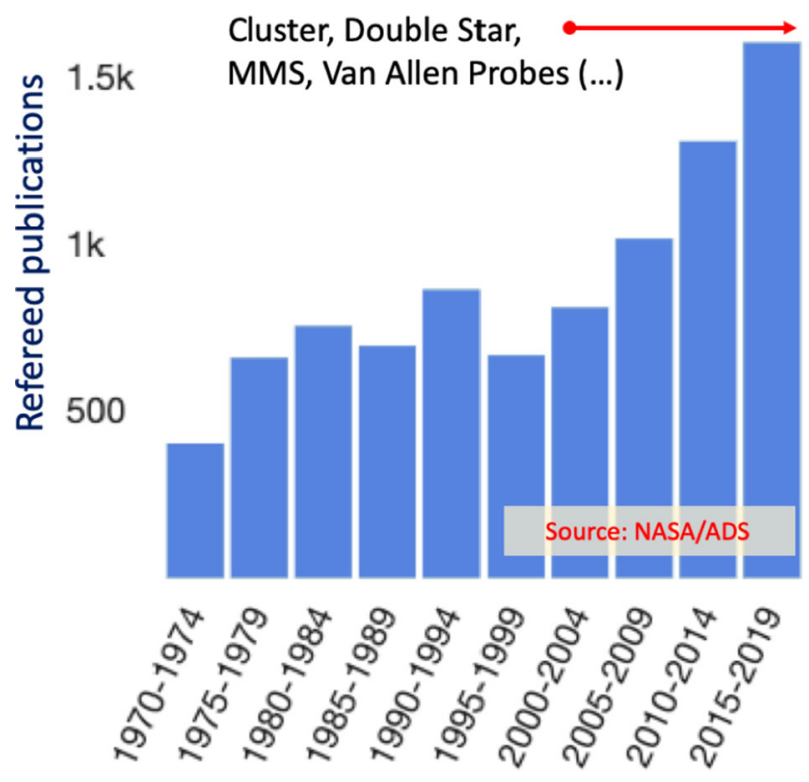

Fig. 11 Number of refereed publications on energetic charged particles and/or the radiation belts of Earth between 1970 and 2019 from https://ui.adsabs.harvard.edu/. Created with the command: abs:( ((radiation belt) OR (energetic particles)) NOT Jupiter NOT Saturn NOT Uranus NOT Mars NOT Mercury NOT Neptune AND Earth) AND year:1970-2019

generated covering many years and different radiation belts, enabling detailed comparative studies for the first time. Planned or ongoing missions would add to that: the limits of radiation belt formation can be exploited with BepiColombo at the weakly magnetized Mercury, and with JUICE at Ganymede's mini-magnetosphere [49]. Potential extension of such observations to Uranus or Neptune [52, 74] would allow us to explore radiation belts across a variety of environments and scales. Xray telescopes, following their application on the SMILE and BepiColombo missions $[14,21]$, will become readily available for Jupiter spacecraft and open a new era in the exploration of planetary magnetospheres.

\subsection{Opportunities for interdisciplinary science investigations}

Magnetospheric and Jupiter system science A mission designed for radiation belt studies is suited to explore any other magnetospheric region it crosses. The mission concepts considered (Section 3.3) are sufficient to extend and/or complement many of the magnetospheric science goals of Galileo, Juno, JUICE, and Europa Clipper [6, 61, 127]. The option for coordinated, multi-point measurements could be a first for a giant planet's magnetosphere, with obvious advantages (Section 4.2). The strong coupling between the radiation belts and Jupiter offers many opportunities to study different components of the jovian system, besides what is described in Section 2.6. For instance, Juno data show that Jupiter's internally generated magnetic field has changed in the time between Pioneer 10 and 11 observations and today $[32,114]$. 
The observed variations, attributed to advection of the field by Jupiter's zonal winds, could be further monitored with a radiation belt mission reaching to low jovian altitudes two decades after Juno. Potential close moon flybys offer a chance to conduct new type of measurements: the detection of high energy albedo particles from moon surfaces, released due to the precipitation of very high energy ions, is an example. Such particles, recently detected at Earth's Moon, offer insights on the physical properties of the immediate subsurface $[148,176]$.

Heliospheric physics ENA imaging offers opportunities to explore the formation, interactions, and the large-scale structure of the global heliosphere, providing insights on the plasma processes at $\sim 100 \mathrm{AU}$ and beyond. Relevant investigations by the Interstellar Boundary Explorer (IBEX) and Cassini/INCA have already revolutionized our notions about the global heliosphere [42, 82, 103], established an increasingly growing community and new missions either in preparation (IMAP [104]) or in consideration (Interstellar Probe [106]). Potential measurements by an ENA imager at Jupiter would extend the time history of previous measurements. Regular GCR observations and a detailed view of escaping relativistic electrons from Jupiter, offered by the mission's energetic particle detectors, when combined with similar observations at $1 \mathrm{AU}$, would update models of particle transport in the heliosphere [170].

Astrophysics Jupiter offers many astrophysical parallels (Section 1.3). While detections of magnetized exoplanets are increasing [114], no exoplanetary radiation belt synchrotron emissions have been detected, possibly due to their weak intensity and the large distance from Earth. Predictions about the radiation environment of exoplanets may thus rely on our understanding of radiation belts in our Solar System. Radiation belts can be part of any strongly magnetized object, not just of (exo)planets. Ultra-cool brown dwarfs (UCBD) appear to host magnetospheres resembling those of our Solar System. Radio emissions of the UCBD NLTT 33370 B not only have similarities to those emitted from Jupiter's radiation belts, but also seem to indicate that the object's magnetosphere has a magnetodisc-like configuration with a dense plasma torus, another striking similarity with the giant planet $[174,175]$. Understanding the processes at work in Jupiter's radiation belt can also inform the study of rotating radio transients which may originate from particle scattering within pulsar radiation belts [89]. Finally, in-situ measurements at Jupiter offer insights on how a strong charged particle accelerator can seed its surrounding environment with particle radiation. The study of Jovian Cosmic Rays, i.e. energetic electrons and ions escaping into the heliosphere $[91,170]$, in conjunction with similar studies at other planets $[83,94]$ could update models of how this escape develops with implications about "local cosmic ray" sources in different astrophysical systems.

Radiation belt environment models Jupiter's mini-Solar System is a very attractive target for many exploration missions. For any of these, considerations of Jupiter's radiation environment are always a high priority. Radiation environment models, bound to the existing data limitations, have uncertainties (e.g. [48]) that could propagate into mission design in the form of extra shielding mass (or less payload mass). 
A comprehensive in-situ investigation of Jupiter's radiation belts would support empirical radiation belt model design and offer opportunities to optimize the future exploration of the jovian system and many of its rings and moons are contained within its harshest energetic particle environment.

\section{Summary}

Jupiter's radiation belts are a major component of the planet's magnetosphere, but the unprecedented scale, complexity and energetics make their measurement very demanding. Their severe energetic particle environment has challenged, and still challenges, their in-situ spacecraft exploration. However, the outstanding science that can be performed through their in-situ study should motivate us to overcome these hurdles and plan for their comprehensive survey. The Juno mission has passed repeatedly through Jupiter's belts and has shown this to be a feasible technical task. Experience gained from the JUICE mission, and the highly anticipated results of its scientific investigations, will pave the way for the next step in the exploration of the jovian system by ESA. In this article, originally a White Paper submitted in response to the European Space Agency's call for science themes for its Voyage 2050 programme, we have argued why a multi-spacecraft mission to Jupiter's radiation belts should be that next step. A first multi-spacecraft mission to a giant planet's magnetosphere and radiation belts lends itself to numerous important discoveries, as the extraordinary success of ESA's Cluster mission, a similar "first", has proven. The interdisciplinary character of the investigations that can be performed in Jupiter's system will only increase the mission's impact further. We therefore believe that Jupiter's radiation belts deserve to be assigned as a high priority target in the Voyage 2050 programme cycle and we urge ESA to conduct and support the necessary mission, instrument, and technology studies that would eventually make their in-situ exploration a reality.

\section{Appendix 1: Full list of coauthors and endorsers}

This article is based on a White Paper submitted in response to the European Space Agency's call for science themes for its Voyage 2050 programme. In addition to the 29 authors of the White Paper, many more scientists have contributed to and/or endorsed its content and that of a similar White Paper [119] since its original submission. The full list of 97 scientists from 16 countries is provided below in alphabetical order:

1. P. Addison, Georgia Institute of Technology, Atlanta, GA, USA

2. O. Allanson, University of Exeter, UK

3. L. Alves, Instituto Nacional de Pesquisas Espaciais, Sao Jose dos Campos, Brazil

4. N. André, Institut de Recherche en Astrophysique et Planétologie, CNRS, CNES, Université de Toulouse, Toulouse, France 
5. L. Arruda, LIP, Lisbon, Portugal

6. B. Bertucci, University of Perugia, Perugia, Italy

7. L. Blum, University of Colorado, Boulder, CO, USA

8. S. Bourdarie, ONERA, Toulouse, France

9. P. Brandt, John Hopkins University/Applied Physics Laboratory, Laurel, MD, USA

10. G. Branduardi-Raymont, UCL Mullard Space Science Laboratory, Dorking, UK,

11. B. R. Breer, Georgia Institute of Technology, Atlanta, GA, USA

12. A. Brunet, ONERA, Toulouse, France

13. E. Bunce, University of Leicester, Leicester, UK

14. X. Cao, University of Iowa, Iowa City, IA, USA

15. G. Clark, John Hopkins University/Applied Physics Laboratory, Laurel, MD, USA

16. C. Cohen, California Institute of Technology, Pasadena, CA, USA

17. I. Cohen, John Hopkins University/Applied Physics Laboratory, Laurel, MD, USA

18. J. F. Cooper, Emeritus, NASA Goddard Space Flight Center, Greenbelt, MD, USA

19. I.A. Daglis, National and Kapodistrian University of Athens, Athens, Greece

20. I. Dandouras, Institut de Recherche en Astrophysique et Planétologie, CNRS, CNES, Université de Toulouse, Toulouse, France

21. L. Da Silva, State Key Laboratory of Space Weather, National Space Science Center, Chinese Academy of Sciences, China and Instituto Nacional de Pesquisas Espaciais, Sao Jose dos Campos, Brazil

22. I. de Pater, UC Berkeley, Berkeley, CA, USA

23. M. de Souza Franco, Instituto Nacional de Pesquisas Espaciais, Sao Jose dos Campos, Brazil

24. R. T. Desai, Imperial College London, London, UK

25. K. Dialynas, Academy of Athens, Athens, Greece

26. A. Drozdov, UCLA, Los Angeles, CA, USA

27. W. Dunn, UCL Mullard Space Science Laboratory, Dorking, UK

28. E. Echer, Instituto Nacional de Pesquisas Espaciais, Sao Jose dos Campos, Brazil

29. S. Fatemi, Department of Physics at Umeå University, Umeå, Sweden

30. C. Forsyth, UCL Mullard Space Science Laboratory, Dorking, UK

31. Y. Futaana, Swedish Institute of Space Physics (IRF), Kiruna, Sweden

32. H. B. Garrett, NASA Jet Propulsion Laboratory, Pasadena, CA, USA

33. M. Gkioulidou, John Hopkins University/Applied Physics Laboratory, Laurel, MD, USA

34. P. Gonçalves, LIP, Lisbon, Portugal

35. Y. Hao, Peking university, Peking, China

36. M. Hedman, University of Idaho, Moscow, ID, USA

37. R. B. Horne, British Antarctic Survey, Cambridge, UK

38. G. Hospodarsky, University of Iowa, Iowa City, IA, USA 
39. H. Huybrighs, ESA/ESTEC, Noordwijk, Netherlands

40. C. M. Jackman, Dublin Institute for Advanced Studies, Dublin, Ireland

41. A. N. Jaynes, University of Iowa, Iowa City, IA, USA

42. X. Jia, University of Michigan, Ann Arbor, MI, USA

43. G. H. Jones, UCL Mullard Space Science Laboratory, Dorking, UK

44. I. Jun, NASA Jet Propulsion Laboratory, Pasadena, CA, USA

45. T. Keane, NASA Jet Propulsion Laboratory, Pasadena, CA, USA

46. K. Khurana, UCLA, Los Angeles, CA, USA

47. H. Kita, Tohoku Institute of Technology, Sendai, Japan

48. P. Kollmann, John Hopkins University/Applied Physics Laboratory, Laurel, MD, USA

49. A. Kotova, Institut de Recherche en Astrophysique et Planétologie, CNRS, CNES, Université de Toulouse, Toulouse, France

50. R. Kraft, Center for Astrophysics, Harvard and Smithsonian, Cambridge, MA, USA

51. E. A. Kronberg, Ludwig Maximilian University of Munich, Munich, Germany

52. N. Krupp, Max Planck Institute for Solar System Research, Göttingen, Germany

53. S. Lejosne, UC Berkeley, Berkeley, CA, USA

54. W. Li, Center for Space Physics, Boston University, Boston, Massachusetts, USA

55. X. Li, LASP and AES, University of Colorado, Boulder, CO, USA

56. L. Liuzzo, UC Berkeley, Berkeley, CA, USA

57. V. Maget, ONERA, Toulouse, France

58. P. Marchezi, Instituto Nacional de Pesquisas Espaciais, Sao Jose dos Campos, Brazil

59. R. Marshall, University of Colorado, Boulder, CO, USA

60. B. H. Mauk, John Hopkins University/Applied Physics Laboratory, Laurel, MD, USA

61. C. Moeckel, UC Berkeley, Berkeley, CA, USA

62. G. Murakami, JAXA/ISAS, Sagamihara, Kanagawa, Japan

63. Q. Nénon, ESA-ESTEC, Noordwijk, The Netherlands

64. T. A. Nordheim, NASA Jet Propulsion Laboratory, Pasadena, CA, USA

65. B. Palmaerts, University of Liege, Liege, Belgium

66. C. Paranicas, John Hopkins University/Applied Physics Laboratory, Laurel, MD, USA

67. C. Paty, University of Oregon, Eugene, OR, USA

68. M. Pinto, LIP, Lisbon, Portugal

69. C. Plainaki, Agenzia Spaziale Italiana (ASI), Rome, Italy

70. A. R. Poppe, Space Sciences Laboratory, University of California at Berkeley

71. J. Rae, Northumbria University, Newcastle, UK

72. L. H. Regoli, John Hopkins University/Applied Physics Laboratory, Laurel, MD, USA

73. E. Roussos, Max Planck Institute for Solar System Research, Göttingen, Germany 
74. O. Santolik, Institute of Atmospheric Physics of the Czech Academy of Sciences, Prague, Czechia and Faculty of Mathematics and Physics, Charles University, Prague, Czechia

75. D. Santos-Costa, SwRI, San Antonio, TX, USA

76. T. Sarris, Department of Electrical and Computer Engineering, Democritus University of Thrace, Xanthi, Greece

77. Q. Q. Shi, Space Science Institute, School of Space Science and Physics, Shandong University, Shandong, China

78. A. Sicard, ONERA, Toulouse, France

79. S. Simon, Georgia Institute of Technology, Atlanta, GA, USA

80. H. T. Smith, John Hopkins University/Applied Physics Laboratory, Laurel, MD, USA

81. Y. Y. Shprits, German Research Center for Geoscience, GFZ-Potsdam, Potsdam, Germany and Institute for Physics and Astrophysics, University of Potsdam, Potsdam, Germany

82. K. Sorathia, John Hopkins University/Applied Physics Laboratory, Laurel, MD, USA

83. A. Sulaiman, University of Iowa, Iowa City, IA, USA

84. Y. Sun, Peking university, Peking, China

85. J. R. Szalay, Princeton University, Princeton, NJ, USA

86. D. L. Turner, John Hopkins University/Applied Physics Laboratory, Laurel, MD, USA

87. S. Ukhorskiy, John Hopkins University/Applied Physics Laboratory, Laurel, MD, USA

88. E. A. Vieira, Instituto Nacional de Pesquisas Espaciais, Sao Jose dos Campos, Brazil

89. D. Wang, German Research Center for Geoscience, GFZ-Potsdam, Potsdam, Germany

90. Y. Wei, of Geology and Geophysics, Chinese Academy of Sciences, Beijing, China

91. P. K. G. Williams, Center for Astrophysics, Harvard and Smithsonian, Cambridge, MA, USA and American Astronomical Society, Washington, DC, USA

92. E. E. Woodfield, British Antarctic Survey, Cambridge, UK

93. X. Wu, University of Geneva, Geneva, Switzerland

94. Z. H. Yao, Institute of Geology and Geophysics, Chinese Academy of Sciences, Beijing, China

95. C. Yuan, Institute of Geology and Geophysics, Chinese Academy of Sciences, Beijing, China

96. P. Zarka, LESIA, CNRS, Observatoire de Paris, PSL, Meudon, France

97. Q. G. Zong, Peking university, Peking, China

Funding Open Access funding enabled and organized by Projekt DEAL.

\section{Declarations}

Conflict of Interests The authors declare that they have no conflict of interest. 
Open Access This article is licensed under a Creative Commons Attribution 4.0 International License, which permits use, sharing, adaptation, distribution and reproduction in any medium or format, as long as you give appropriate credit to the original author(s) and the source, provide a link to the Creative Commons licence, and indicate if changes were made. The images or other third party material in this article are included in the article's Creative Commons licence, unless indicated otherwise in a credit line to the material. If material is not included in the article's Creative Commons licence and your intended use is not permitted by statutory regulation or exceeds the permitted use, you will need to obtain permission directly from the copyright holder. To view a copy of this licence, visit http://creativecommons.org/licenses/by/4.0/.

\section{References}

1. Alfonsi, L., Ambroglini, F., Ambrosi, G., Ammendola, R., Assante, D., Badoni, D., Belyaev, V.A., Burger, W.J., Cafagna, A., Cipollone, P., Consolini, G., Conti, L., Contin, A., Angelis, E.D., Donato, C.D., Franceschi, G.D., Santis, A.D., Santis, C.D., Diego, P., Durante, M., Fornaro, C., Guandalini, C., Laurenti, G., Laurenza, M., Lazzizzera, I., Lolli, M., Manea, C., Marcelli, L., Marcucci, F., Masciantonio, G., Osteria, G., Palma, F., Palmonari, F., Panico, B., Patrizii, L., Picozza, P., Pozzato, M., Rashevskaya, I., Ricci, M., Rovituso, M., Scotti, V., Sotgiu, A., Sparvoli, R., Spataro, B., Spogli, L., Tommasino, F., Ubertini, P., Vannaroni, G., Xuhui, S., Zoffoli, S., Cses-Limadou Collaboration: The HEPD particle detector and the EFD electric field detector for the CSES satellite. Radiat. Phys. Chem. 137, 187-192 (2017). https://doi.org/10.1016/j.radphyschem.2016.12.022

2. Anglin, J.D., Burrows, J.R., Mu, J.L., Wilson, M.D.: Trapped energetic ions in Jupiter's inner magnetosphere. J. Geophys. Res. 102(A1), 1-36 (1997). https://doi.org/10.1029/96JA02681

3. Atwell, W., Townsend, L., Miller, T., Campbell, C.: A reassessment of Galileo radiation exposures in the Jupiter magnetosphere. Radiat. Prot. Dosim. 116(1-4), 220-223 (2005). https://doi.org/10.1093/rpd/nci009

4. Atzei, A., Wielders, A., Stankov, A., Falkner, P.: Overview of the esa jovian technology reference studies. https://sci.esa.int/web/future-missions-department/-/40866-jovian-studies-overview (2007)

5. Bagenal, F.: The magnetosphere of Jupiter: Coupling the equator to the poles. J. Atmos. Sol. Terr. Phys. 69(3), 387-402 (2007). https://doi.org/10.1016/j.jastp.2006.08.012

6. Bagenal, F., Adriani, A., Allegrini, F., Bolton, S.J., Bonfond, B., Bunce, E.J., Connerney, J.E.P., Cowley, S.W.H., Ebert, R.W., Gladstone, G.R., Hansen, C.J., Kurth, W.S., Levin, S.M., Mauk, B.H., McComas, D.J., Paranicas, C.P., Santos-Costa, D., Thorne, R.M., Valek, P., Waite, J.H., Zarka, P.: Magnetospheric science objectives of the Juno mission. Space Sci. Rev. 213(1-4), 219-287 (2017). https://doi.org/10.1007/s11214-014-0036-8

7. Bagenal, F., Wilson, R.J., Siler, S., Paterson, W.R., Kurth, W.S.: Survey of Galileo plasma observations in Jupiter's plasma sheet. J. Geophys. Res. Planets 121(5), 871-894 (2016). https://doi.org/10.1002/2016JE005009

8. Baker, D.N., Erickson, P.J., Fennell, J.F., Foster, J.C., Jaynes, A.N., Verronen, P.T.: Space weather effects in the Earth's radiation belts. Space Sci. Rev. 214(1), 17 (2018). https://doi.org/10.1007/s11214-017-0452-7

9. Baker, D.N., Jaynes, A.N., Hoxie, V.C., Thorne, R.M., Foster, J.C., Li, X., Fennell, J.F., Wygant, J.R., Kanekal, S.G., Erickson, P.J., Kurth, W., Li, W., Ma, Q., Schiller, Q., Blum, L., Malaspina, D.M., Gerrard, A., Lanzerotti, L.J.: An impenetrable barrier to ultrarelativistic electrons in the Van Allen radiation belts. Nature 515(7528), 531-534 (2014). https://doi.org/10.1038/nature13956

10. Baker, D.N., Panasyuk, M.I.: Discovering Earth's radiation belts. Phys. Today 70(12), 46-51 (2017). https://doi.org/10.1063/PT.3.3791

11. Barbosa, D.D., Eviatar, A., Siscoe, G.L.: On the acceleration of energetic ions in Jupiter's magnetosphere. J. Geophys. Res. 89(A6), 3789-3800 (1984). https://doi.org/10.1029/JA089iA06p03789

12. Becker, H.N., Alexander, J.W., Adriani, A., Mura, A., Cicchetti, A., Noschese, R., Jørgensen, J.L., Denver, T., Sushkova, J., Jørgensen, A., Benn, M., Connerney, J.E.P., Bolton, S.J., Allison, J., Watts, S., Adumitroaie, V., Manor-Chapman, E.A., Daubar, I.J., Lee, C., Kang, S., McAlpine, W.J., Di Iorio, T., Pasqui, C., Barbis, A., Lawton, P., Spalsbury, L., Loftin, S., Sun, J.: The Juno radiation monitoring (RM) investigation. Space Sci. Rev. 213(1-4), 507-545 (2017). https://doi.org/10.1007/s11214-017-0345-9 
13. Becker, H.N., Santos-Costa, D., Jørgensen, J.L., Denver, T., Adriani, A., Mura, A., Connerney, J.E.P., Bolton, S.J., Levin, S.M., Thorne, R.M., Alexander, J.W., Adumitroaie, V., Manor-Chapman, E.A., Daubar, I.J., Lee, C., Benn, M., Sushkova, J., Cicchetti, A., Noschese, R.: Observations of $\mathrm{MeV}$ electrons in Jupiter's innermost radiation belts and polar regions by the Juno radiation monitoring investigation: Perijoves 1 and 3. Geophys. Res. Lett. 44(10), 4481-4488 (2017). https://doi.org/10.1002/2017GL073091

14. Benkhoff, J., van Casteren, J., Hayakawa, H., Fujimoto, M., Laakso, H., Novara, M., Ferri, P., Middleton, H.R., Ziethe, R.: BepiColombo-Comprehensive exploration of Mercury: Mission overview and science goals. Planet. Space Sci. 58(1-2), 2-20 (2010). https://doi.org/10.1016/j.pss.2009.09.020

15. Birmingham, T.J.: Charged particle motions in the distended magnetosphere of Jupiter and Saturn. J. Geophys. Res. 87(A9), 7421-7430 (1982). https://doi.org/10.1029/JA087iA09p07421

16. Blake, J.B., Carranza, P.A., Claudepierre, S.G., Clemmons, J.H., Crain, W.R., Dotan, Y., Fennell, J.F., Fuentes, F.H., Galvan, R.M., George, J.S., Henderson, M.G., Lalic, M., Lin, A.Y., Looper, M.D., Mabry, D.J., Mazur, J.E., McCarthy, B., Nguyen, C.Q., O’Brien, T.P., Perez, M.A., Redding, M.T., Roeder, J.L., Salvaggio, D.J., Sorensen, G.A., Spence, H.E., Yi, S., Zakrzewski, M.P.: The magnetic electron ion spectrometer (MagEIS) instruments aboard the radiation belt storm probes (RBSP) spacecraft. Space Sci. Rev. 179(1-4), 383-421 (2013). https://doi.org/10.1007/s11214-013-9991-8

17. Bolton, S.J., Janssen, M., Thorne, R., Levin, S., Klein, M., Gulkis, S., Bastian, T., Sault, R., Elachi, C., Hofstadter, M., Bunker, A., Dulk, G., Gudim, E., Hamilton, G., Johnson, W.T.K., Leblanc, Y., Liepack, O., McLeod, R., Roller, J., Roth, L., West, R.: Ultra-relativistic electrons in Jupiter's radiation belts. Nature 415(6875), 987-991 (2002). https://doi.org/10.1038/415987a

18. Bolton, S.J., Lunine, J., Stevenson, D., Connerney, J.E.P., Levin, S., Owen, T.C., Bagenal, F., Gautier, D., Ingersoll, A.P., Orton, G.S., Guillot, T., Hubbard, W., Bloxham, J., Coradini, A., Stephens, S.K., Mokashi, P., Thorne, R., Thorpe, R.: The Juno mission. Space Sci. Rev. 213(1-4), 5-37 (2017). https://doi.org/10.1007/s11214-017-0429-6

19. Bonfond, B., Gladstone, G.R., Grodent, D., Gérard, J.C., Greathouse, T.K., Hue, V., Kammer, J.A., Versteeg, M.H., Davis, M.W., Becker, H.N., Radioti, A., Elliott, S.S., Imai, M., Paranicas, C.P., Bolton, S.J., Levin, S.M., Connerney, J.E.P.: Bar code events in the Juno-UVS data: Signature of $10 \mathrm{MeV}$ electron Microbursts at Jupiter. Geophys. Res. Lett. 45(22), 12,108-12,115 (2018). https://doi.org/10.1029/2018GL080490

20. Brandt, P.C., Hsieh, S.Y., DeMajistre, R., Mitchell, D.G., Marghitu, O., Wheatland, M.: ENA imaging of planetary ring currents. In: Keiling, A. (ed.) Electric Currents in Geospace and Beyond, vol. 235, pp. 93-114 (2018). https://doi.org/10.1002/9781119324522.ch6

21. Branduardi-Raymont, G., Sembay, S., Carter, J., Ezoe, Y.: Exploring geospace via solar wind charge exchange X-rays. In: European Planetary Science Congress, pp. EPSC2018-937 (2018)

22. Brice, N., Mcdonough, T.R.: Jupiter's radiation belts. Icarus 18(2), 206-219 (1973). https://doi.org/10.1016/0019-1035(73)90204-2

23. Carbary, J.F., Mitchell, D.G., Brandt, P., Roelof, E.C., Krimigis, S.M.: Statistical morphology of ENA emissions at Saturn. J. Geophys. Res. Space Phys. 113(A5), A05210 (2008). https://doi.org/10.1029/2007JA012873

24. Carlson, R.W., Anderson, M.S., Johnson, R.E., Smythe, W.D., Hendrix, A.R., Barth, C.A., Soderblom, L.A., Hansen, G.B., McCord, T.B., Dalton, J.B., Clark, R.N., Shirley, J.H., Ocampo, A.C., Matson, D.L.: Hydrogen Peroxide on the Surface of Europa. Science 283, 2062 (1999). https://doi.org/10.1126/science.283.5410.2062

25. CAS: http://english.cas.cn/newsroom/archive/news_archive/nu2017/201709/t20170908_182912. shtml (2017)

26. Chyba, C.F.: Energy for microbial life on Europa. Nature 403(6768), 381-382 (2000). https://doi.org/10.1038/35000281

27. Clark, G., Mauk, B.H., Haggerty, D., Paranicas, C., Kollmann, P., Rymer, A., Bunce, E.J., Cowley, S.W.H., Mitchell, D.G., Provan, G., Ebert, R.W., Allegrini, F., Bagenal, F., Bolton, S., Connerney, J., Kotsiaros, S., Kurth, W.S., Levin, S., McComas, D.J., Saur, J., Valek, P.: Energetic particle signatures of magnetic field-aligned potentials over Jupiter's polar regions. Geophys. Res. Lett. 44(17), 87038711 (2017). https://doi.org/10.1002/2017GL074366

28. Clark, G., Mauk, B.H., Paranicas, C., Kollmann, P., Smith, H.T.: Charge states of energetic oxygen and sulfur ions in Jupiter's magnetosphere. J. Geophys. Res. Space Phys. 121(3), 2264-2273 (2016). https://doi.org/10.1002/2015JA022257 
29. Clark, G., Tao, C., Mauk, B.H., Nichols, J., Saur, J., Bunce, E.J., Allegrini, F., Gladstone, R., Bagenal, F., Bolton, S., Bonfond, B., Connerney, J., Ebert, R.W., Gershman, D.J., Haggerty, D., Kimura, T., Kollmann, P., Kotsiaros, S., Kurth, W.S., Levin, S., McComas, D.J., Murakami, G., Paranicas, C., Rymer, A., Valek, P.: Precipitating electron energy flux and characteristic energies in Jupiter's main auroral region as measured by Juno/JEDI. J. Geophys. Res. Space Phys. 123(9), 7554-7567 (2018). https://doi.org/10.1029/2018JA025639

30. Cohen, C.M.S., Garrard, T.L., Stone, E.C., Cooper, J.F., Murphy, N., Gehrels, N.: Io encounters past and present: A heavy ion comparison. J. Geophys. Res. 105(A4), 7775-7782 (2000). https://doi.org/10.1029/1999JA000021

31. Cohen, C.M.S., Stone, E.C., Selesnick, R.S.: Energetic ion observations in the middle Jovian magnetosphere. J. Geophys. Res. 106(A12), 29871-29882 (2001). https://doi.org/10.1029/2001JA000008

32. Connerney, J.E.P., Kotsiaros, S., Oliversen, R.J., Espley, J.R., Joergensen, J.L., Joergensen, P.S., Merayo, J.M.G., Herceg, M., Bloxham, J., Moore, K.M., Bolton, S.J., Levin, S.M.: A New Model of Jupiter's Magnetic Field From Juno's First Nine Orbits. Geophys. Res. Lett. 45(6), 2590-2596 (2018). https://doi.org/10.1002/2018GL077312

33. Cooper, J.F.: Nuclear cascades in Saturn's rings: Cosmic ray albedo neutron decay and origins of trapped protons in the inner magnetosphere. J. Geophys. Res. 88(A5), 3945-3954 (1983). https://doi.org/10.1029/JA088iA05p03945

34. Cooper, J.F., Sturner, S.J.: Energetic radiation from galactic cosmic ray interactions with Saturn's main rings. J. Geophys. Res. Space Phys. 123(9), 7473-7485 (2018). https://doi.org/10.1029/2018JA025583

35. de Pater, I., Dunn, D.E.: VLA observations of Jupiter's synchrotron radiation at 15 and $22 \mathrm{GHz}$. Icarus 163(2), 449-455 (2003). https://doi.org/10.1016/S0019-1035(03)00068-X

36. de Pater, I., Goertz, C.K.: Radial diffusion models of energetic electrons and Jupiter's synchrotron radiation. 1. Steady state solution. J. Geophys. Res. 95, 39-50 (1990). https://doi.org/10.1029/JA095iA01p00039

37. de Pater, I., Goertz, C.K.: Radial diffusion models of energetic electrons and Jupiter's synchrotron radiation. 2. Time variability. J. Geophys. Res. 99(A2), 2271-2288 (1994). https://doi.org/10.1029/93JA02097

38. Dialynas, K., Brandt, P.C., Krimigis, S.M., Mitchell, D.G., Hamilton, D.C., Krupp, N., Rymer, A.M.: The extended Saturnian neutral cloud as revealed by global ENA simulations using Cassini/MIMI measurements. J. Geophys. Res. Space Phys. 118(6), 3027-3041 (2013). https://doi.org/10.1002/jgra.50295

39. Dodge, R., Boyles, M., Rasbach, C.: Key and driving requirements for the Juno payload suite of instruments. https://doi.org/10.2514/6.2007-6111. https://arc.aiaa.org/doi/abs/10.2514/6.2007-6111 (2012)

40. Dougherty, L.P., Bodisch, K.M., Bagenal, F.: Survey of Voyager plasma science ions at Jupiter: 2. Heavy ions. J. Geophys. Res. Space Phys. 122(8), 8257-8276 (2017). https://doi.org/10.1002/ 2017JA024053

41. Dumont, M., Grodent, D., Radioti, A., Bonfond, B., Gérard, J.C.: Jupiter's equatorward auroral features: Possible signatures of magnetospheric injections. J. Geophys. Res. Space Phys. 119(12), 10,068-10,077 (2014). https://doi.org/10.1002/2014JA020527

42. Dunn, W.R., Branduardi-Raymont, G., Ray, L.C., Jackman, C.M., Kraft, R.P., Elsner, R.F., Rae, I.J., Yao, Z., Vogt, M.F., Jones, G.H., Gladstone, G.R., Orton, G.S., Sinclair, J.A., Ford, P.G., Graham, G.A., Caro-Carretero, R., Coates, A.J.: The independent pulsations of Jupiter's northern and southern X-ray auroras. Nature Astron. 1, 758-764 (2017). https://doi.org/10.1038/s41550-017-0262-6

43. Ebert, R.W., Allegrini, F., Bagenal, F., Beebe, C., Dayeh, M.A., Desai, M.I., George, D., Hanley, J., Mokashi, P., Murphy, N., Valek, P.W., Wenkert, D., Wolf, A., Yen, C.W.L.: JUpiter MagnetosPheric boundary ExploreR (JUMPER). In: Lunar and Planetary Science Conference, Lunar and Planetary Science Conference, p. 1284 (2018)

44. Ebert, R.W., Greathouse, T.K., Clark, G., Allegrini, F., Bagenal, F., Bolton, S.J., Connerney, J.E.P., Gladstone, G.R., Imai, M., Hue, V., Kurth, W.S., Levin, S., Louarn, P., Mauk, B.H., McComas, D.J., Paranicas, C., Szalay, J.R., Thomsen, M.F., Valek, P.W., Wilson, R.J.: Comparing electron energetics and UV brightness in Jupiter's Northern Polar region during Juno Perijove 5. Geophys. Res. Lett. 46(1), 19-27 (2019). https://doi.org/10.1029/2018GL081129

45. Elsner, R.F., Gladstone, G.R., Waite, J.H., Crary, F.J., Howell, R.R., Johnson, R.E., Ford, P.G., Metzger, A.E., Hurley, K.C., Feigelson, E.D., Garmire, G.P., Bhardwaj, A., Grodent, D.C., Majeed, T., 
Tennant, A.F., Weisskopf, M.C.: Discovery of soft X-Ray emission from Io, Europa, and the Io plasma torus. ApJ 572(2), 1077-1082 (2002). https://doi.org/10.1086/340434

46. ESA: Juice industry day, “exploring the emergence of habitable worlds around gas giants". http:// emits.sso.esa.int/emits-doc/ESTEC/JUICE-Industry-Day-booklet_v2.pdf (2015)

47. Escoubet, C.P., Masson, A., Laakso, H., Goldstein, M.L.: Recent highlights from Cluster, the first 3-D magnetospheric mission. Annales Geophysicae 33(10), 1221-1235 (2015). https://doi.org/10.5194/angeo-33-1221-2015

48. Evans, H.D.R., Daly, E.J., Nieminen, P., Santin, G., Erd, C.: Jovian radiation belt models, uncertainties and margins. IEEE Trans. Nucl. Sci. 60(4), 2397-2403 (2013). https://doi.org/10.1109/TNS.2013.2249097

49. Eviatar, A., Williams, D.J., Paranicas, C., McEntire, R.W., Mauk, B.H., Kivelson, M.G.: Trapped Energetic electrons in the magnetosphere of Ganymede. J. Geophys. Res. 105(A3), 5547-5554 (2000). https://doi.org/10.1029/1999JA900450

50. Fieseler, P.D., Ardalan, S.M., Frederickson, A.R.: The radiation effects on galileo spacecraft systems at Jupiter. IEEE Trans. Nucl. Sci. 49(6), 2739-2758 (2002). https://doi.org/10.1109/TNS.2002.805386

51. Fischer, H.M., Pehlke, E., Wibberenz, G., Lanzerotti, L.J., Mihalov, J.D.: High-energy charged particles in the innermost Jovian magnetosphere. Science 272(5263), 856-858 (1996). https://doi.org/10.1126/science.272.5263.856

52. Fletcher, L.N., Helled, R., Roussos, E., Jones, G., Charnoz, S., AndrÃ®e, N., Andrews, D., Bannister, M., Bunce, E., CavaliÃ@), T., Ferri, F., Fortney, J., Grassi, D., Griton, L., Hartogh, P., Hueso, R., Kaspi, Y., Lamy, L., Masters, A., Melin, H., Moses, J., Mousis, O., Nettleman, N., Plainaki, C., Schmidt, J., Simon, A., Tobie, G., Tortora, P., Tosi, F., Turrini, D.: Ice giant systems: The scientific potential of orbital missions to uranus and neptune. Planet. Space Sci., 105030. https://doi.org/10.1016/j.pss.2020.105030. http://www.sciencedirect. com/science/article/pii/S0032063320300040 (2020)

53. Fritz, T.A.: The cusp as a source of magnetospheric energetic particles, currents, and electric fields: a new paradigm. Space Sci. Rev. 95, 469-488 (2001)

54. Galopeau, P.H.M., Gérard, E.: Variations of Jupiter's synchrotron radiation: a link with solar activity? Planet. Space Sci. 49(13), 1379-1391 (2001). https://doi.org/10.1016/S0032-0633(01)00066-6

55. Ganushkina, N.Y., Dandouras, I., Shprits, Y.Y., Cao, J.: Locations of boundaries of outer and inner radiation belts as observed by Cluster and Double Star. J. Geophys. Res. Space Phys. 116(A9), A09234 (2011). https://doi.org/10.1029/2010JA016376

56. Garrard, T.L., Stone, E.C., Murphy, N.: Effects of absorption by lo on composition of energetic heavy lons. Science 274(5286), 393-394 (1996). https://doi.org/10.1126/science.274.5286.393

57. Garrett, H., Martinez-Sierra, L.M., Evans, R.: Updating the jovian proton radiation environment 2015. https://ntrs.nasa.gov/search.jsp?R=20160009376 (2015)

58. Garrett, H.B., Kim, W., Evans, R.W.: Updating the jovian plasma and radiation environments: The latest results for 2015. J. Spacecr. Rocket. 53(4), 693-707 (2016). https://doi.org/10.2514/1.A33510

59. Gehrels, N., Stone, E.C.: Energetic oxygen and sulfur ions in the Jovian magnetosphere and their contribution to the auroral excitation. J. Geophys. Res. 88(A7), 5537-5550 (1983). https://doi.org/10.1029/JA088iA07p05537

60. Glass, G., Jain, M., Evans, M.L., Hiebert, J.C., Northcliffe, L.C., Bonner, B.E., Simmons, J.E., Bjork, C., Riley, P., Cassapakis, C.: Neutron spectra at $0^{\circ}$ from proton-proton collisions between 647 and 805 MeV. Phys. Rev. D 15(1), 36-46 (1977). https://doi.org/10.1103/PhysRevD.15.36

61. Grasset, O., Dougherty, M.K., Coustenis, A., Bunce, E.J., Erd, C., Titov, D., Blanc, M., Coates, A., Drossart, P., Fletcher, L.N., Hussmann, H., Jaumann, R., Krupp, N., Lebreton, J.P., PrietoBallesteros, O., Tortora, P., Tosi, F., Van Hoolst, T.: JUpiter ICy moons Explorer (JUICE): An ESA mission to orbit Ganymede and to characterise the Jupiter system. Planet. Space Sci. 78, 1-21 (2013). https://doi.org/10.1016/j.pss.2012.12.002

62. Green, J.C., Kivelson, M.G.: Relativistic electrons in the outer radiation belt: Differentiating between acceleration mechanisms. J. Geophys. Res. Space Phys. 109(A3), A03213 (2004). https://doi.org/10.1029/2003JA010153

63. Haggerty, D.K., Mauk, B.H., Paranicas, C.P., Clark, G., Kollmann, P., Rymer, A.M., Bolton, S.J., Connerney, J.E.P., Levin, S.M.: Juno/JEDI observations of 0.01 to $<10 \mathrm{MeV}$ energetic ions in the Jovian auroral regions: Anticipating a source for polar X-ray emission. Geophys. Res. Lett. 44(13), 6476-6482 (2017). https://doi.org/10.1002/2017GL072866 
64. Halekas, J.S., Angelopoulos, V., Sibeck, D.G., Khurana, K.K., Russell, C.T., Delory, G.T., Farrell, W.M., McFadden, J.P., Bonnell, J.W., Larson, D., Ergun, R.E., Plaschke, F., Glassmeier, K.H.: First results from ARTEMIS, a new two-spacecraft lunar mission: counterstreaming plasma populations in the lunar wake. Space Sci. Rev. 165(1-4), 93-107 (2011). https://doi.org/10.1007/s11214-010-9738-8

65. Han, S., Murakami, G., Kita, H., Tsuchiya, F., Tao, C., Misawa, H., Yamazaki, A., Nakamura, M.: Investigating solar wind-driven electric field influence on long-term dynamics of jovian synchrotron radiation. J. Geophys. Res. Space Phys. 123(11), 9508-9516 (2018). https://doi.org/10.1029/2018JA025849

66. Hess, W.N., Birmingham, T.J., Mead, G.D.: Absorption of trapped particles by Jupiter's moons. J. Geophys. Res. 79(19), 2877 (1974). https://doi.org/10.1029/JA079i019p02877

67. Hess, W.N., Patterson, H.W., Wallace, R., Chupp, E.L.: Cosmic-ray neutron energy spectrum. Phys. Rev. 116(2), 445-457 (1959). https://doi.org/10.1103/PhysRev.116.445

68. Horne, R.B., Thorne, R.M., Shprits, Y.Y., Meredith, N.P., Glauert, S.A., Smith, A.J., Kanekal, S.G., Baker, D.N., Engebretson, M.J., Posch, J.L., Spasojevic, M., Inan, U.S., Pickett, J.S., Decreau, P.M.E.: Wave acceleration of electrons in the Van Allen radiation belts. Nature 437(7056), 227-230 (2005). https://doi.org/10.1038/nature03939

69. Jones, G.H., Snodgrass, C., Wicks, R.T.: A proposed ESA mission to a dynamically new comet. In: AGU Fall meeting abstracts, pp. P54D-15 (2018)

70. Kennel, C.F., Coroniti, F.V.: Is Jupiter's magnetosphere like a pulsar's or Earth's? Space Sci. Rev. 17(6), 857-883 (1975). https://doi.org/10.1007/BF00777259

71. Kim, T.K., Ebert, R.W., Valek, P.W., Allegrini, F., McComas, D.J., Bagenal, F., Connerney, J.E.P., Livadiotis, G., Thomsen, M.F., Wilson, R.J., Bolton, S.J.: Survey of ion properties in Jupiter's plasma sheet: Juno JADE-I observations. J. Geophys. Res. Space Phys. 125(4), e27696 (2020). https://doi.org/10.1029/2019JA027696

72. Kita, H., Misawa, H., Bhardwaj, A., Tsuchiya, F., Murakami, G., Tao, C., Kimura, T., Yoshioka, K., Yamazaki, A., Kasaba, Y., Yoshikawa, I., Fujimoto, M.: Short-term Variation in the dawn-dusk asymmetry of the Jovian radiation belt obtained from GMRT and Hisaki EXCEED observations. ApJ 872(2), L24 (2019). https://doi.org/10.3847/2041-8213/ab0427

73. Kita, H., Misawa, H., Tsuchiya, F., Tao, C., Morioka, A.: Effect of solar UV/EUV heating on the intensity and spatial distribution of Jupiter's synchrotron radiation. J. Geophys. Res. Space Phys. 118(10), 6106-6115 (2013). https://doi.org/10.1002/jgra.50568

74. Kollmann, P., Cohen, I., Allen, R.C., Clark, G., Roussos, E., Vines, S., Dietrich, W., Wicht, J., de Pater, I., Runyon, K.D., Cartwright, R., Masters, A., Brain, D., Hibbits, K., Mauk, B., Gkioulidou, M., Rymer, A., McNutt, R., Hue, V., Stanley, S., Brandt, P.: Magnetospheric studies: a requirement for addressing interdisciplinary mysteries in the ice giant systems. Space Sci. Rev. 216(5), 78 (2020). https://doi.org/10.1007/s11214-020-00696-5

75. Kollmann, P., Paranicas, C., Clark, G., Mauk, B.H., Haggerty, D.K., Rymer, A.M., Santos-Costa, D., Connerney, J.E.P., Allegrini, F., Valek, P., Kurth, W.S., Gladstone, G.R., Levin, S., Bolton, S.: A heavy ion and proton radiation belt inside of Jupiter's rings. Geophys. Res. Lett. 44(11), 5259-5268 (2017). https://doi.org/10.1002/2017GL073730

76. Kollmann, P., Paranicas, C., Clark, G., Roussos, E., Lagg, A., Krupp, N.: The vertical thickness of Jupiter's Europa gas torus from charged particle measurements. Geophys. Res. Lett. 43(18), 94259433 (2016). https://doi.org/10.1002/2016GL070326

77. Kollmann, P., Roussos, E., Kotova, A., Paranicas, C., Krupp, N.: The evolution of Saturn's radiation belts modulated by changes in radial diffusion. Nature Astron. 1, 872-877 (2017). https://doi.org/10.1038/s41550-017-0287-X

78. Kollmann, P., Roussos, E., Kotova, A., Regoli, L., Mitchell, D.G., Carbary, J., Clark, G., Krupp, N., Paranicas, C.: Saturn's innermost radiation belt throughout and inward of the d-ring. Geophys. Res. Lett. 45(20), 10,912-10,920 (2018). https://doi.org/10.1029/2018GL077954

79. Kollmann, P., Roussos, E., Paranicas, C., Woodfield, E.E., Mauk, B.H., Clark, G., Smith, D.C., Vandegriff, J.: Electron acceleration to MeV energies at Jupiter and Saturn. J. Geophys. Res. Space Phys. 123(11), 9110-9129 (2018). https://doi.org/10.1029/2018JA025665

80. Kotova, A., Roussos, E., Kollmann, P., Krupp, N., Dand ouras, I.: Galactic cosmic rays access to the magnetosphere of Saturn. J. Geophys. Res. Space Phys. 124(1), 166-177 (2019). https://doi.org/10.1029/2018JA025661

81. Krimigis, S.M., Mitchell, D.G., Hamilton, D.C., Livi, S., Dandouras, J., Jaskulek, S., Armstrong, T.P., Boldt, J.D., Cheng, A.F., Gloeckler, G., Hayes, J.R., Hsieh, K.C., Ip, W.H., Keath, E.P., 
Kirsch, E., Krupp, N., Lanzerotti, L.J., Lundgren, R., Mauk, B.H., McEntire, R.W., Roelof, E.C., Schlemm, C.E., Tossman, B.E., Wilken, B., Williams, D.J.: Magnetosphere imaging instrument (MIMI) on the Cassini mission to Saturn/Titan. Space Sci. Rev. 114(1-4), 233-329 (2004). https://doi.org/10.1007/s11214-004-1410-8

82. Krimigis, S.M., Mitchell, D.G., Roelof, E.C., Hsieh, K.C., McComas, D.J.: Imaging the interaction of the heliosphere with the interstellar medium from Saturn with Cassini. Science 326(5955), 971 (2009). https://doi.org/10.1126/science.1181079

83. Krimigis, S.M., Sergis, N., Dialynas, K., Mitchell, D.G., Hamilton, D.C., Krupp, N., Dougherty, M., Sarris, E.T.: Analysis of a sequence of energetic ion and magnetic field events upstream from the Saturnian magnetosphere. Planet. Space Sci. 57(14-15), 1785-1794 (2009). https://doi.org/10.1016/j.pss.2009.02.013

84. Kronberg, E.A., Glassmeier, K.H., Woch, J., Krupp, N., Lagg, A., Dougherty, M.K.: A possible intrinsic mechanism for the quasi-periodic dynamics of the Jovian magnetosphere. J. Geophys. Res. Space Phys. 112(A5), A05203 (2007). https://doi.org/10.1029/2006JA011994

85. Li, X.: High quality sciences by CubeSat missions and future potential. In: EGU General Assembly Conference Abstracts, EGU General Assembly Conference Abstracts, p. 9982 (2019)

86. Li, X., Selesnick, R., Schiller, Q., Zhang, K., Zhao, H., Baker, D.N., Temerin, M.A.: Measurement of electrons from albedo neutron decay and neutron density in near-Earth space. Nature 552(7685), 382-385 (2017). https://doi.org/10.1038/nature24642

87. Lillis, R.J., Barjatya, A., Curtis, D.W., Curry, S., Parker, J., Parrish, N., Russell, C.T., Larson, D.E., Livi, R., Bester, M., Brain, D., Ma, Y., Modolo, R., Harada, Y., Fowler, C.M., Luhmann, J.G., Williams, A.: The ESCAPADE mission to Mars: Escape and plasma acceleration and dynamics explorers. In: AGU Fall Meeting Abstracts, vol. 2018, pp. P54D-04 (2018)

88. Louarn, P., Paranicas, C.P., Kurth, W.S.: Global magnetodisk disturbances and energetic particle injections at Jupiter. J. Geophys. Res. Space Phys. 119(6), 4495-4511 (2014). https://doi.org/10.1002/2014JA019846

89. Luo, Q., Melrose, D.: Pulsar radiation belts and transient radio emission. MNRAS 378(4), 14811490 (2007). https://doi.org/10.1111/j.1365-2966.2007.11889.x

90. Ma, Q., Li, W., Zhang, X.J., Bagenal, F.: Energetic electron scattering due to whistler mode chorus waves using realistic magnetic field and density models in jupiter's magnetosphere. J. Geophys. Res. Space Phys, e2020JA027968. https://doi.org/10.1029/2020JA027968

91. Marhavilas, P.K., Anagnostopoulos, G.C., Sarris, E.T.: Periodic signals in Ulysses' energetic particle events upstream and downstream from the Jovian bow shock. Planet. Space Sci. 49(10-11), 10311047 (2001). https://doi.org/10.1016/S0032-0633(01)00018-6

92. Mauk, B.H.: Comparative investigation of the energetic ion spectra comprising the magnetospheric ring currents of the solar system. J. Geophys. Res. Space Phys. 119(12), 9729-9746 (2014). https://doi.org/10.1002/2014JA020392

93. Mauk, B.H., Clark, G., Allegrini, F., Bagenal, F., Bolton, S.J., Connerney, J.E.P., Haggerty, D.K., Kollmann, P., Mitchell, D.G., Paranicas, C.P., Rymer, A.M.: Juno Energetic Neutral Atom (ENA) Remote Measurements of Magnetospheric Injection Dynamics in Jupiter's Io Torus Regions. J. Geophys. Res. Space Phys. 125(5), e27964 (2020). https://doi.org/10.1029/2020JA027964

94. Mauk, B.H., Cohen, I.J., Haggerty, D.K., Hospodarsky, G.B., Connerney, J.E.P., Anderson, B.J., Bagenal, F., Ebert, R.W., Bolton, S.J., Burch, J.L., Levin, S.M., Torbert, R.B., Vines, S.K., Westlake, J.H.: Investigation of Mass-/Charge-Dependent Escape of Energetic Ions Across the Magnetopauses of Earth and Jupiter. J. Geophys. Res. Space Phys. 124(7), 5539-5567 (2019). https://doi.org/10.1029/2019JA026626

95. Mauk, B.H., Fox, N.J.: Electron radiation belts of the solar system. J. Geophys. Res. Space Phys. 115(A12), A12220 (2010). https://doi.org/10.1029/2010JA015660

96. Mauk, B.H., Haggerty, D.K., Jaskulek, S.E., Schlemm, C.E., Brown, L.E., Cooper, S.A., Gurnee, R.S., Hammock, C.M., Hayes, J.R., Ho, G.C., Hutcheson, J.C., Jacques, A.D., Kerem, S., Kim, C.K., Mitchell, D.G., Nelson, K.S., Paranicas, C.P., Paschalidis, N., Rossano, E., Stokes, M.R.: The Jupiter energetic particle detector instrument (JEDI) investigation for the Juno mission. Space Sci. Rev. 213(1-4), 289-346 (2017). https://doi.org/10.1007/s11214-013-0025-3

97. Mauk, B.H., Haggerty, D.K., Paranicas, C., Clark, G., Kollmann, P., Rymer, A.M., Bolton, S.J., Levin, S.M., Adriani, A., Allegrini, F., Bagenal, F., Bonfond, B., Connerney, J.E.P., Gladstone, G.R., Kurth, W.S., McComas, D.J., Valek, P.: Discrete and broadband electron acceleration in Jupiter's powerful aurora. Nature 549(7670), 66-69 (2017). https://doi.org/10.1038/nature23648 
98. Mauk, B.H., Haggerty, D.K., Paranicas, C., Clark, G., Kollmann, P., Rymer, A.M., Mitchell, D.G., Bolton, S.J., Levin, S.M., Adriani, A., Allegrini, F., Bagenal, F., Connerney, J.E.P., Gladstone, G.R., Kurth, W.S., McComas, D.J., Ranquist, D., Szalay, J.R., Valek, P.: Juno observations of energetic charged particles over Jupiter's polar regions: Analysis of monodirectional and bidirectional electron beams. Geophys. Res. Lett. 44(10), 4410-4418 (2017). https://doi.org/10.1002/2016GL072286

99. Mauk, B.H., Haggerty, D.K., Paranicas, C., Clark, G., Kollmann, P., Rymer, A.M., Peachey, J.M., Bolton, S.J., Levin, S.M., Adriani, A., Allegrini, F., Bagenal, F., Bonfond, B., Connerney, J.E.P., Ebert, R.W., Gladstone, G.R., Kurth, W.S., McComas, D.J., Ranquist, D., Valek, P.: Diverse electron and ion acceleration characteristics observed over Jupiter's main aurora. Geophys. Res. Lett. 45(3), 1277-1285 (2018). https://doi.org/10.1002/2017GL076901

100. Mauk, B.H., Mitchell, D.G., Krimigis, S.M., Roelof, E.C., Paranicas, C.P.: Energetic neutral atoms from a trans-Europa gas torus at Jupiter. Nature 421(6926), 920-922 (2003). https://doi.org/10.1038/nature01431

101. Mauk, B.H., Saur, J.: Equatorial electron beams and auroral structuring at Jupiter. J. Geophys. Res. Space Phys. 112(A10), A10221 (2007). https://doi.org/10.1029/2007JA012370

102. McComas, D.J., Alexander, N., Allegrini, F., Bagenal, F., Beebe, C., Clark, G., Crary, F., Desai, M.I., De Los Santos, A., Demkee, D., Dickinson, J., Everett, D., Finley, T., Gribanova, A., Hill, R., Johnson, J., Kofoed, C., Loeffler, C., Louarn, P., Maple, M., Mills, W., Pollock, C., Reno, M., Rodriguez, B., Rouzaud, J., Santos-Costa, D., Valek, P., Weidner, S., Wilson, P., Wilson, R.J., White, D.: The Jovian auroral distributions experiment (JADE) on the Juno mission to Jupiter. Space Sci. Rev. 213(1-4), 547-643 (2017). https://doi.org/10.1007/s11214-013-9990-9

103. McComas, D.J., Allegrini, F., Bochsler, P., Bzowski, M., Christian, E.R., Crew, G.B., DeMajistre, R., Fahr, H., Fichtner, H., Frisch, P.C., Funsten, H.O., Fuselier, S.A., Gloeckler, G., Gruntman, M., Heerikhuisen, J., Izmodenov, V., Janzen, P., Knappenberger, P., Krimigis, S., Kucharek, H., Lee, M., Livadiotis, G., Livi, S., MacDowall, R.J., Mitchell, D., Möbius, E., Moore, T., Pogorelov, N.V., Reisenfeld, D., Roelof, E., Saul, L., Schwadron, N.A., Valek, P.W., Vanderspek, R., Wurz, P., Zank, G.P.: Global observations of the interstellar interaction from the interstellar boundary explorer (IBEX). Science 326(5955), 959 (2009). https://doi.org/10.1126/science.1180906

104. McComas, D.J., Christian, E.R., Schwadron, N.A., Fox, N., Westlake, J., Allegrini, F., Baker, D.N., Biesecker, D., Bzowski, M., Clark, G., Cohen, C.M.S., Cohen, I., Dayeh, M.A., Decker, R., de Nolfo, G.A., Desai, M.I., Ebert, R.W., Elliott, H.A., Fahr, H., Frisch, P.C., Funsten, H.O., Fuselier, S.A., Galli, A., Galvin, A.B., Giacalone, J., Gkioulidou, M., Guo, F., Horanyi, M., Isenberg, P., Janzen, P., Kistler, L.M., Korreck, K., Kubiak, M.A., Kucharek, H., Larsen, B.A., Leske, R.A., Lugaz, N., Luhmann, J., Matthaeus, W., Mitchell, D., Moebius, E., Ogasawara, K., Reisenfeld, D.B., Richardson, J.D., Russell, C.T., Sokół, J.M., Spence, H.E., Skoug, R., Sternovsky, Z., Swaczyna, P., Szalay, J.R., Tokumaru, M., Wiedenbeck, M.E., Wurz, P., Zank, G.P., Zirnstein, E.J.: Interstellar mapping and acceleration probe (IMAP): A new NASA mission. Space Sci. Rev. 214(8), 116 (2018). https://doi.org/10.1007/s11214-018-0550-1

105. McKibben, R.B., Simpson, J.A., Zhang, M.: Impulsive bursts of relativistic electrons discovered during Ulysses' traversal of Jupiter's dusk-side magnetosphere. Planet. Space Sci. 41(11-12), 10411058 (1993). https://doi.org/10.1016/0032-0633(93)90108-E

106. McNutt, R.L., Wimmer-Schweingruber, R.F., Gruntman, M., Krimigis, S.M., Roelof, E.C., Brandt, P.C., Vernon, S.R., Paul, M.V., Lathrop, B.W., Mehoke, D.S., Napolillo, D.H., Stough, R.W.: Near-term interstellar probe: First step. Acta Astronaut. 162, 284-299 (2019). https://doi.org/10.1016/j.actaastro.2019.06.013

107. Menietti, J.D., Groene, J.B., Averkamp, T.F., Horne, R.B., Woodfield, E.E., Shprits, Y.Y., SoriaSantacruz Pich, M., Gurnett, D.A.: Survey of whistler mode chorus intensity at Jupiter. J. Geophys. Res. Space Phys. 121(10), 9758-9770 (2016). https://doi.org/10.1002/2016JA022969

108. Menietti, J.D., Shprits, Y.Y., Horne, R.B., Woodfield, E.E., Hospodarsky, G.B., Gurnett, D.A.: Chorus, $\mathrm{ECH}$, and $\mathrm{Z}$ mode emissions observed at Jupiter and Saturn and possible electron acceleration. J. Geophys. Res. Space Phys. 117(A12), A12214 (2012). https://doi.org/10.1029/2012JA018187

109. Michel, F.C.: The astrophysics of Jupiter. Space Sci. Rev. 24(4), 381-406 (1979). https://doi.org/10.1007/BF00172210

110. Milillo, A., Plainaki, C., De Angelis, E., Mangano, V., Massetti, S., Mura, A., Orsini, S., Rispoli, R.: Analytical model of Europa's $\mathrm{O}_{2}$ exosphere. Planet. Space Sci. 130, 3-13 (2016). https://doi.org/10.1016/j.pss.2015.10.011 
111. Mitchell, D.G., Brandt, P.C., Westlake, J.H., Jaskulek, S.E., Andrews, G.B., Nelson, K.S.: Energetic particle imaging: The evolution of techniques in imaging high-energy neutral atom emissions. J. Geophys. Res. Space Phys. 121(9), 8804-8820 (2016). https://doi.org/10.1002/2016JA022586

112. Miyoshi, Y., Shinohara, I., Takashima, T., Asamura, K., Higashio, N., Mitani, T., Kasahara, S., Yokota, S., Kazama, Y., Wang, S.Y., Tam, S.W.Y., Ho, P.T.P., Kasahara, Y., Kasaba, Y., Yagitani, S., Matsuoka, A., Kojima, H., Katoh, Y., Shiokawa, K., Seki, K.: Geospace exploration project ERG. Earth, Planets, and Space 70(1), 101 (2018). https://doi.org/10.1186/s40623-018-0862-0

113. Moeckel, C., Janssen, M., de Pater, I.: A re-analysis of the Jovian radio emission as seen by Cassini-RADAR and evidence for time variability. Icarus 321, 994-1012 (2019). https://doi.org/10.1016/j.icarus.2018.12.013

114. Moore, K.M., Cao, H., Bloxham, J., Stevenson, D.J., Connerney, J.E.P., Bolton, S.J.: Time variation of Jupiter's internal magnetic field consistent with zonal wind advection. Nature Astron. 3, 730-735 (2019). https://doi.org/10.1038/s41550-019-0772-5

115. Murakami, G., Yoshioka, K., Yamazaki, A., Tsuchiya, F., Kimura, T., Tao, C., Kita, H., Kagitani, M., Sakanoi, T., Uemizu, K., Kasaba, Y., Yoshikawa, I., Fujimoto, M.: Response of Jupiter's inner magnetosphere to the solar wind derived from extreme ultraviolet monitoring of the Io plasma torus. Geophys. Res. Lett. 43(24), 12,308-12,316 (2016). https://doi.org/10.1002/2016GL071675

116. NASA/JPL: Europa clipper newsletter. https:/europa.nasa.gov/system/internal_resources/details/ original/116_Europa-Clipper-Newsletter-External-vol2issue1_FINAL.pdf (2018)

117. Nénon, Q.: Study and modelisation of the Jupiter's radiation belts. Theses, Doctorat de l'Université de Toulouse délivré par l'Institut Supérieur de l'Aéronautique et de 1'Espace (ISAE). https://hal. archives-ouvertes.fr/tel-01899128 (2018)

118. Nénon, Q., André, N.: Evidence of Europa neutral gas torii from energetic sulfur ion measurements. Geophys. Res. Lett. 46(7), 3599-3606 (2019). https://doi.org/10.1029/2019GL082200

119. Nenon, Q., Clark, G., Jun, I., Kollmann, P., Liuzzo, L., Mauk, B., Nordheim, T.A., Poppe, A.R., Roussos, E., Shprits, Y.Y., Turner, D.L., Woodfield, E.E.: Open science questions and missing measurements in the radiation belts of Jupiter. In: Bulletin of the American Astronomical Society, vol. 53, p. 088 (2021). https://doi.org/10.3847/25c2cfeb.fb50005f

120. Nénon, Q., Sicard, A., Bourdarie, S.: A new physical model of the electron radiation belts of Jupiter inside Europa's orbit. J. Geophys. Res. Space Phys. 122(5), 5148-5167 (2017). https://doi.org/10.1002/2017JA023893

121. Nénon, Q., Sicard, A., Caron, P.: The rings of jupiter as seen by the electron and proton radiation belt model Salammbô. Geophys. Res. Lett. 45(20), 10,838-10,846 (2018). https://doi.org/10.1029/2018GL080157

122. Nénon, Q., Sicard, A., Kollmann, P., Garrett, H.B., Sauer, S.P.A., Paranicas, C.: A physical model of the proton radiation belts of Jupiter inside Europa's orbit. J. Geophys. Res. Space Phys. 123(5), 3512-3532 (2018). https://doi.org/10.1029/2018JA025216

123. Nordheim, T.A., Hand, K.P., Paranicas, C.: Preservation of potential biosignatures in the shallow subsurface of Europa. Nature Astron. 2, 673-679 (2018). https://doi.org/10.1038/s41550-018-0499-8

124. Nordheim, T.A., Jasinski, J.M., Hand, K.P.: Galactic cosmic-ray bombardment of Europa's surface. ApJ 881(2), L29 (2019). https://doi.org/10.3847/2041-8213/ab3661

125. Numazawa, M., Ezoe, Y., Ishikawa, K., Ohashi, T., Miyoshi, Y., Kimura, T., Uchiyama, Y., Shiota, D., Brand uardi-Raymont, G.: Suzaku observation of Jupiter's X-rays around solar maximum. PASJ 71(5), 93 (2019). https://doi.org/10.1093/pasj/psz077

126. Ozeke, L.G., Mann, I.R., Murphy, K.R., Degeling, A.W., Claudepierre, S.G., Spence, H.E.: Explaining the apparent impenetrable barrier to ultra-relativistic electrons in the outer Van Allen belt. Nat. Commun. 9, 1844 (2018). https://doi.org/10.1038/s41467-018-04162-3

127. Pappalardo, R.T., Senske, D.A., Korth, H., Klima, R., Vance, S.D., Craft, K.: The Europa clipper mission: Exploring the habitability of a unique icy world. In: European Planetary Science Congress, pp. EPSC2017-304 (2017)

128. Paranicas, C., Hibbitts, C.A., Kollmann, P., Ligier, N., Hendrix, A.R., Nordheim, T.A., Roussos, E., Krupp, N., Blaney, D., Cassidy, T.A., Clark, G.: Magnetospheric considerations for solar system ice state. Icarus 302, 560-564 (2018). https://doi.org/10.1016/j.icarus.2017.12.013

129. Paranicas, C., Mauk, B.H., Haggerty, D.K., Clark, G., Kollmann, P., Rymer, A.M., Bonfond, B., Dunn, W.R., Ebert, R.W., Gladstone, G.R., Roussos, E., Krupp, N., Bagenal, F., Levin, S.M., Connerney, J.E.P., Bolton, S.J.: Intervals of intense energetic electron beams over Jupiter's poles. J. Geophys. Res. Space Phys. 123(3), 1989-1999 (2018). https://doi.org/10.1002/2017JA025106 
130. Paranicas, C., Mauk, B.H., Haggerty, D.K., Clark, G., Kollmann, P., Rymer, A.M., Westlake, J., Allen, R.C., Szalay, J., Ebert, R.W., Sulaiman, A.H., Imai, M., Roussos, E., Krupp, N., Nénon, Q., Bagenal, F., Bolton, S.J.: Io's effect on energetic charged particles as seen in juno data. Geophys. Res. Lett. 46(23), 13,615-13,620 (2019). https://doi.org/10.1029/2019GL085393

131. Paranicas, C., Mauk, B.H., Khurana, K., Jun, I., Garrett, H., Krupp, N., Roussos, E.: Europa's near-surface radiation environment. Geophys. Res. Lett. 34(15), L15103 (2007). https://doi.org/10.1029/2007GL030834

132. Picozza, P., Galper, A.M., Castellini, G., Adriani, O., Altamura, F., Ambriola, M., Barbarino, G.C., Basili, A., Bazilevskaja, G.A., Bencardino, R., Boezio, M., Bogomolov, E.A., Bonechi, L., Bongi, M., Bongiorno, L., Bonvicini, V., Cafagna, F., Campana, D., Carlson, P., Casolino, M., de Marzo, C., de Pascale, M.P., de Rosa, G., Fedele, D., Hofverberg, P., Koldashov, S.V., Krutkov, S.Y., Kvashnin, A.N., Lund, J., Lundquist, J., Maksumov, O., Malvezzi, V., Marcelli, L., Menn, W., Mikhailov, V.V., Minori, M., Misin, S., Mocchiutti, E., Morselli, A., Nikonov, N.N., Orsi, S., Osteria, G., Papini, P., Pearce, M., Ricci, M., Ricciarini, S.B., Runtso, M.F., Russo, S., Simon, M., Sparvoli, R., Spillantini, P., Stozhkov, Y.I., Taddei, E., Vacchi, A., Vannuccini, E., Voronov, S.A., Yurkin, Y.T., Zampa, G., Zampa, N., Zverev, V.G.: PAMELA A payload for antimatter matter exploration and light-nuclei astrophysics. Astropart. Phys. 27(4), 296-315 (2007). https://doi.org/10.1016/j.astropartphys.2006.12.002

133. Pinto, M., Goncalves, P., Marques, A., Pinto, J.C., Hajdas, W.: Development of a Directionality Detector for RADEM, the Radiation Hard Electron Monitor Aboard the JUICE Mission. IEEE Trans. Nucl. Sci. 66(7), 1770-1777 (2019). https://doi.org/10.1109/TNS.2019.2900398

134. Plainaki, C., Cassidy, T.A., Shematovich, V.I., Milillo, A., Wurz, P., Vorburger, A., Roth, L., Galli, A., Rubin, M., Blöcker, A., Brandt, P.C., Crary, F., Dandouras, I., Jia, X., Grassi, D., Hartogh, P., Lucchetti, A., McGrath, M., Mangano, V., Mura, A., Orsini, S., Paranicas, C., Radioti, A., Retherford, K.D., Saur, J., Teolis, B.: Towards a global unified model of Europa's tenuous atmosphere. Space Sci. Rev. 214(1), 40 (2018). https://doi.org/10.1007/s11214-018-0469-6

135. Plainaki, C., Lilensten, J., Radioti, A., Andriopoulou, M., Milillo, A., Nordheim, T.A., Dand ouras, I., Coustenis, A., Grassi, D., Mangano, V., Massetti, S., Orsini, S., Lucchetti, A.: Planetary space weather: scientific aspects and future perspectives. J. Space Weather. Space Clim. 6, A31 (2016). https://doi.org/10.1051/swsc/2016024

136. Podzolko, M.V., Getselev, I.V., Gubar', Y.I., Veselovsky, I.S.: Radiation conditions of a mission to Jupiter and Europa. Sol. Syst. Res. 43(2), 116-120 (2009). https://doi.org/10.1134/S00380946090 20038

137. Potgieter, M., Nndanganeni, R.: The combined modulation of Jovian and Galactic electrons in the heliosphere. In: 35th International Cosmic Ray Conference (ICRC2017), International Cosmic Ray Conference, vol. 301, p. 43 (2017)

138. Roussos, E., Jones, G.H., Krupp, N., Paranicas, C., Mitchell, D.G., Lagg, A., Woch, J., Motschmann, U., Krimigis, S.M., Dougherty, M.K.: Electron microdiffusion in the Saturnian radiation belts: Cassini MIMI/LEMMS observations of energetic electron absorption by the icy moons. J. Geophys. Res. Space Phys. 112(A6), A06214 (2007). https://doi.org/10.1029/2006JA012027

139. Roussos, E., Kollmann, P.: The Radiation Belts of Jupiter and Saturn, chap. 32, pp. 499-514. American Geophysical Union (AGU), Washington (2021). https://doi.org/10.1002/9781119815624.ch32

140. Roussos, E., Kollmann, P., Krupp, N., Kotova, A., Regoli, L., Paranicas, C., Mitchell, D.G., Krimigis, S.M., Hamilton, D., Brandt, P., Carbary, J., Christon, S., Dialynas, K., Dand ouras, I., Hill, M.E., Ip, W.H., Jones, G.H., Livi, S., Mauk, B.H., Palmaerts, B., Roelof, E.C., Rymer, A., Sergis, N., Smith, H.T.: A radiation belt of energetic protons located between Saturn and its rings, vol. 362. https://doi.org/10.1126/science.aat1962 (2018)

141. Roussos, E., Kollmann, P., Krupp, N., Paranicas, C., Dialynas, K., Sergis, N., Mitchell, D.G., Hamilton, D.C., Krimigis, S.M.: Drift-resonant, relativistic electron acceleration at the outer planets: Insights from the response of Saturn's radiation belts to magnetospheric storms. Icarus 305, 160-173 (2018). https://doi.org/10.1016/j.icarus.2018.01.016

142. Roussos, E., Krupp, N., Armstrong, T.P., Paranicas, C., Mitchell, D.G., Krimigis, S.M., Jones, G.H., Dialynas, K., Sergis, N., Hamilton, D.C.: Discovery of a transient radiation belt at Saturn. Geophys. Res. Lett. 35(22), L22106 (2008). https://doi.org/10.1029/2008GL035767

143. Roussos, E., Krupp, N., Mitchell, D.G., Paranicas, C., Krimigis, S.M., Andriopoulou, M., Palmaerts, B., Kurth, W.S., Badman, S.V., Masters, A., Dougherty, M.K.: Quasi-periodic injections of relativistic electrons in Saturn's outer magnetosphere. Icarus 263, 101-116 (2016). https://doi.org/10.1016/j.icarus.2015.04.017 
144. Roussos, E., Krupp, N., Paranicas, C., Carbary, J.F., Kollmann, P., Krimigis, S.M., Mitchell, D.G.: The variable extension of Saturn's electron radiation belts. Planet. Space Sci. 104, 3-17 (2014). https://doi.org/10.1016/j.pss.2014.03.021

145. Santolík, O., Gurnett, D.A., Jones, G.H., Schippers, P., Crary, F.J., Leisner, J.S., Hospodarsky, G.B., Kurth, W.S., Russell, C.T., Dougherty, M.K.: Intense plasma wave emissions associated with Saturn's moon Rhea. Geophys. Res. Lett. 38(19), L19204 (2011). https://doi.org/10.1029/2011GL049219

146. Santos-Costa, D., Adumitroaie, V., Ingersoll, A., Gulkis, S., Janssen, M.A., Levin, S.M., Oyafuso, F., Brown, S., Williamson, R., Bolton, S.J., Connerney, J.E.P.: First look at Jupiter's synchrotron emission from Juno's perspective. Geophys. Res. Lett. 44(17), 8676-8684 (2017). https://doi.org/10.1002/2017GL072836

147. Santos-Costa, D., Bolton, S.J., Thorne, R.M., Miyoshi, Y., Levin, S.M.: Investigating the origins of the Jovian decimetric emission's variability. J. Geophys. Res. Space Phys. 113(A1), A01204 (2008). https://doi.org/10.1029/2007JA012396

148. Schwadron, N.A., Wilson, J.K., Looper, M.D., Jordan, A.P., Spence, H.E., Blake, J.B., Case, A.W., Iwata, Y., Kasper, J.C., Farrell, W.M., Lawrence, D.J., Livadiotis, G., Mazur, J., Petro, N., Pieters, C., Robinson, M.S., Smith, S., Townsend, L.W., Zeitlin, C.: Signatures of volatiles in the lunar proton albedo. Icarus 273, 25-35 (2016). https://doi.org/10.1016/j.icarus.2015.12.003

149. Selesnick, R.S.: Cosmic ray access to Jupiter's magnetosphere. Geophys. Res. Lett. 29(9), 1298 (2002). https://doi.org/10.1029/2001GL014146

150. Selesnick, R.S., Baker, D.N., Kanekal, S.G., Hoxie, V.C., Li, X.: Modeling the proton radiation belt with Van Allen probes relativistic electron-proton telescope data. J. Geophys. Res. Space Phys. 123(1), 685-697 (2018). https://doi.org/10.1002/2017JA024661

151. Selesnick, R.S., Cohen, C.M.S.: Charge states of energetic ions in Jupiter's radiation belt inferred from absorption microsignatures of Io. J. Geophys. Res. Space Phys. 114(A1), A01207 (2009). https://doi.org/10.1029/2008JA013722

152. Selesnick, R.S., Cohen, C.M.S., Khurana, K.K.: Energetic ion dynamics in Jupiter's plasma sheet. J. Geophys. Res. 106(A9), 18895-18906 (2001). https://doi.org/10.1029/2000JA000242

153. Selesnick, R.S., Cummings, A.C., Cummings, J.R., Mewaldt, R.A., Stone, E.C., von Rosenvinge, T.T.: Geomagnetically trapped anomalous cosmic rays. J. Geophys. Res. 100(A6), 9503-9518 (1995). https://doi.org/10.1029/94JA03140

154. Selesnick, R.S., Looper, M.D., Mewaldt, R.A.: A theoretical model of the inner proton radiation belt. Space Weather 5(4), S04003 (2007). https://doi.org/10.1029/2006SW000275

155. Shprits, Y.Y., Angelopoulos, V., Russell, C.T., Strangeway, R.J., Runov, A., Turner, D., Caron, R., Cruce, P., Leneman, D., Michaelis, I., Petrov, V., Panasyuk, M., Yashin, I., Drozdov, A., Russell, C.L., Kalegaev, V., Nazarkov, I., Clemmons, J.H.: Scientific objectives of electron losses and fields INvestigation onboard lomonosov satellite. Space Sci. Rev. 214(1), 25 (2018). https://doi.org/10.1007/s11214-017-0455-4

156. Shprits, Y.Y., Drozdov, A.Y., Spasojevic, M., Kellerman, A.C., Usanova, M.E., Engebretson, M.J., Agapitov, O.V., Zhelavskaya, I.S., Raita, T.J., Spence, H.E., Baker, D.N., Zhu, H., Aseev, N.A.: Wave-induced loss of ultra-relativistic electrons in the Van Allen radiation belts. Nat. Commun. 7, 12883 (2016). https://doi.org/10.1038/ncomms12883

157. Shprits, Y.Y., Menietti, J.D., Drozdov, A.Y., Horne, R.B., Woodfield, E.E., Groene, J.B., de Soria-Santacruz, M., Averkamp, T.F., Garrett, H., Paranicas, C., Gurnett, D.A.: Strong whistler mode waves observed in the vicinity of Jupiter's moons. Nat. Commun. 9, 3131 (2018). https://doi.org/10.1038/s41467-018-05431-X

158. Shprits, Y.Y., Menietti, J.D., Gu, X., Kim, K.C., Horne, R.B.: Gyroresonant interactions between the radiation belt electrons and whistler mode chorus waves in the radiation environments of Earth, Jupiter, and Saturn: A comparative study. J. Geophys. Res. Space Phys. 117(A11), A11216 (2012). https://doi.org/10.1029/2012JA018031

159. Simpson, J.A., Anglin, J.D., Balogh, A., Burrows, J.R., Cowley, S.W.H., Ferrando, P., Heber, B., Hynds, R.J., Kunow, H., Marsden, R.G., McKibben, R.B., Muller-Mellin, R., Page, D.E., Raviart, A., Sanderson, T.R., Staines, K., Wenzel, K.P., Wilson, M.D., Zhang, M.: Energetic charged-particle phenomena in the Jovian magnetosphere: First results from the ulysses COSPIN collaboration. Science 257(5076), 1543-1550 (1992). https://doi.org/10.1126/science.257.5076.1543

160. Spergel, M.S.: Low order effect of CRAND input in the Jovian atmosphere. Moon 17(2), 123-131 (1977). https://doi.org/10.1007/BF00640903 
161. Stone, E.C., Cummings, A.C., Looper, M.D., Selesnick, R.S., Lal, N., McDonald, F.B., Trainor, J.H., Chenette, D.L.: Energetic charged particles in the magnetosphere of neptune. Science 246(4936), 1489-1494 (1989). https://doi.org/10.1126/science.246.4936.1489

162. Stratton, J.M., Harvey, R.J., Heyler, G.A.: Mission overview for the radiation belt storm probes mission. Space Sci. Rev. 179(1-4), 29-57 (2013). https://doi.org/10.1007/s11214-012-9933-x

163. Sun, T.R., Wang, C., Sembay, S.F., Lopez, R.E., Escoubet, C.P., Branduardi-Raymont, G., Zheng, J.H., Yu, X.Z., Guo, X.C., Dai, L., Liu, Z.Q., Wei, F., Guo, Y.H.: Soft X-ray imaging of the magnetosheath and cusps under different solar wind conditions: mhd simulations. J. Geophys. Res. Space Phys. 124(4), 2435-2450 (2019). https://doi.org/10.1029/2018JA026093

164. Sun, Y.X., Roussos, E., Krupp, N., Zong, Q.G., Kollmann, P., Zhou, X.Z.: Spectral signatures of adiabatic electron acceleration at saturn through corotation drift cancelation. Geophys. Res. Lett. 46(10240), 10:240-10:249 (2019). https://doi.org/10.1029/2019GL084113

165. Szalay, J.R., Allegrini, F., Bagenal, F., Bolton, S.J., Bonfond, B., Clark, G., Connerney, J.E.P., Ebert, R.W., Gershman, D.J., Giles, R.S., Gladstone, G.R., Greathouse, T., Hospodarsky, G.B., Imai, M., Kurth, W.S., Kotsiaros, S., Louarn, P., McComas, D.J., Saur, J., Sulaiman, A.H., Wilson, R.J.: Alfvénic acceleration sustains ganymede's footprint tail aurora. Geophys. Res. Lett. 47(3), e86527 (2020). https://doi.org/10.1029/2019GL086527

166. Tinsley, T., Sarsfield, M., Stephenson, K.: Update on 241 am production for use in radioisotope power systems. In: 2017 IEEE Aerospace Conference, pp. 1-8 (2017)

167. TomáS, A.T., Woch, J., Krupp, N., Lagg, A., Glassmeier, K.H., Kurth, W.S.: Energetic electrons in the inner part of the Jovian magnetosphere and their relation to auroral emissions. J. Geophys. Res. Space Phys. 109(A6), A06203 (2004). https://doi.org/10.1029/2004JA010405

168. Tsuchiya, F., Misawa, H., Imai, K., Morioka, A.: Short-term changes in Jupiter's synchrotron radiation at $325 \mathrm{MHz}$ : Enhanced radial diffusion in Jupiter's radiation belt driven by solar UV/EUV heating. J. Geophys. Res. Space Phys. 116(A9), A09202 (2011). https://doi.org/10.1029/2010JA016303

169. Vance, S.D., Hand, K.P., Pappalardo, R.T.: Geophysical controls of chemical disequilibria in Europa. Geophys. Res. Lett. 43(10), 4871-4879 (2016). https://doi.org/10.1002/2016GL068547

170. Vogt, A., Heber, B., Kopp, A., Potgieter, M.S., Strauss, R.D.: Jovian electrons in the inner heliosphere. Proposing a new source spectrum based on 30 years of measurements. A\&A 613, A28 (2018). https://doi.org/10.1051/0004-6361/201731736

171. Vogt, R.E., Cook, W.R., Cummings, A.C., Garrard, T.L., Gehrels, N., Stone, E.C., Trainor, J.H., Schardt, A.W., Conlon, T., Lal, N., McDonald, F.B.: Voyager 1: Energetic Ions and Electrons in the Jovian Magnetosphere. Science 204(4396), 1003-1007 (1979). https://doi.org/10.1126/science.204. 4396.1003

172. Westlake, J.H., McNutt, R.L., Kasper, J.C., Case, A.W., Rymer, A.M., Stevens, M.L., Jia, X., Paty, C., Khurana, K.K., Kivelson, M.G., Slavin, J.A., Smith, H.T., Korth, H., Krupp, N., Roussous, E., Saur, J.: The Plasma Instrument for Magnetic Sounding (PIMS) for the Europa Mission. In: 3rd International Workshop on Instrumentation for Planetary Mission, vol. 1980, p. 4037 (2016)

173. Williams, D.J., McEntire, R.W., Jaskulek, S., Wilken, B.: The Galileo energetic particles detector. Space Sci. Rev. 60(1-4), 385-412 (1992). https://doi.org/10.1007/BF00216863

174. Williams, P.K.G.: Modeling Jovian magnetospheres beyond the solar system. In: American astronomical society meeting abstracts, vol. 232, p. 303.04 (2018)

175. Williams, P.K.G., Casewell, S.L., Stark, C.R., Littlefair, S.P., Helling, C., Berger, E.: The first millimeter detection of a non-accreting ultracool dwarf. ApJ 815(1), 64 (2015). https://doi.org/10.1088/0004-637X/815/1/64

176. Wilson, J.K., Spence, H.E., Kasper, J., Golightly, M., Bern Blake, J., Mazur, J.E., Townsend, L.W., Case, A.W., Dixon Looper, M., Zeitlin, C., Schwadron, N.A.: The first cosmic ray albedo proton map of the Moon, vol. 117, p. E00H23 (2012). https://doi.org/10.1029/2011JE003921

177. Woodfield, E.E., Horne, R.B., Glauert, S.A., Menietti, J.D., Shprits, Y.Y.: Electron acceleration at Jupiter: input from cyclotron-resonant interaction with whistler-mode chorus waves. Annales Geophysicae 31(10), 1619-1630 (2013). https://doi.org/10.5194/angeo-31-1619-2013

178. Woodfield, E.E., Horne, R.B., Glauert, S.A., Menietti, J.D., Shprits, Y.Y.: The origin of Jupiter's outer radiation belt. J. Geophys. Res. Space Phys. 119(5), 3490-3502 (2014). https://doi.org/10.1002/2014JA019891

179. Woodfield, E.E., Horne, R.B., Glauert, S.A., Menietti, J.D., Shprits, Y.Y., Kurth, W.S.: Formation of electron radiation belts at Saturn by Z-mode wave acceleration. Nat. Commun. 9, 5062 (2018). https://doi.org/10.1038/s41467-018-07549-4 
180. Wu, X., Ambrosi, G., Azzarello, P., Bergmann, B., Bertucci, B., Cadoux, F., Campbell, M., Duranti, M., Ionica, M., Kole, M., Krucker, S., Maehlum, G., Meier, D., Paniccia, M., Pinsky, L., Plainaki, C., Pospisil, S., Stein, T., Thonet, P.A., Tomassetti, N., Tykhonov, A.: Penetrating particle ANalyzer (PAN). Adv. Space Res. 63(8), 2672-2682 (2019). https://doi.org/10.1016/j.asr.2019.01.012

181. Zong, Q.G., Zhou, X.Z., Wang, Y.F., Li, X., Song, P., Baker, D.N., Fritz, T.A., Daly, P.W., Dunlop, M., Pedersen, A.: Energetic electron response to ULF waves induced by interplanetary shocks in the outer radiation belt. J. Geophys. Res. Space Phys. 114(A10), A10204 (2009). https://doi.org/10.1029/2009JA014393

Publisher's note Springer Nature remains neutral with regard to jurisdictional claims in published maps and institutional affiliations. 


\section{Affiliations}

Elias Roussos ${ }^{1}$ (1) . Oliver Allanson ${ }^{2} \cdot$ Nicolas André $^{3} \cdot$ Bruna Bertucci $^{4}$. Graziella Branduardi-Raymont ${ }^{5}$. George Clark $^{6} \cdot$ Konstantinos Dialynas $^{7}$. lannis Dandouras ${ }^{3}$. Ravindra T. Desai ${ }^{8}$. Yoshifumi Futaana ${ }^{9}$. Matina Gkioulidou ${ }^{6}$. Geraint H. Jones ${ }^{5}$. Peter Kollmann ${ }^{6}$. Anna Kotova ${ }^{3}$. Elena A. Kronberg ${ }^{10}$. Norbert Krupp ${ }^{11}$. Go Murakami ${ }^{12}$. Quentin Nénon ${ }^{13}$. Tom Nordheim ${ }^{14}$. Benjamin Palmaerts ${ }^{15}$. Christina Plainaki ${ }^{16}$. Jonathan Rae ${ }^{17}$. Daniel Santos-Costa ${ }^{18}$. Theodore Sarris ${ }^{19} \cdot$ Yuri Shprits ${ }^{20,21}$. Ali Sulaiman ${ }^{22} \cdot$ Emma Woodfield $^{23} \cdot \mathrm{Xin} \mathrm{Wu}^{24} \cdot$ Zonghua $\mathrm{Yao}^{25}$

1 Max Planck Institute for Solar System Research, Justus-von-Liebig-Weg 3, 37077, Göttingen, Germany

2 College of Engineering, Mathematics and Physical Sciences, University of Exeter, Exeter, UK

3 Institut de Recherche en Astrophysique et Planétologie, CNRS, CNES, Université de Toulouse, Toulouse, France

4 University of Perugia, Perugia, Italy

5 UCL Mullard Space Science Laboratory, Dorking, UK

6 Johns Hopkins Applied Physics Laboratory, Laurel, MD, USA

7 Academy of Athens, Athens, Greece

8 Imperial College London, London, UK

9 Swedish Institute for Space Physics (IRF), Kiruna, Sweden

10 Ludwig Maximilian University of Munich, Munich, Germany

11 Max Planck Institute for Solar System Research, Göttingen, Germany

12 Institute of Space and Astronautical Science, JAXA, Kanagawa, Japan

13 ESA-ESTEC, Noordwijk, Netherlands

14 Jet Propulsion Laboratory, Pasadena, CA, USA

15 University of Liege, Liege, Belgium

16 Agenzia Spaziale Italiana (ASI), Rome, Italy

17 Northumbria University, Newcastle, UK

18 Southwest Research Institute, Midwest City, OK, USA

19 Department of Electrical and Computer Engineering, Democritus University of Thrace, Xanthi, Greece

20 German Research Center for Geoscience, GFZ-Potsdam, Potsdam, Germany

21 Institute for Physics and Astrophysics, University of Potsdam, Potsdam, Germany

22 University of Iowa, Iowa City, IA, USA

23 British Antarctic Survey, Cambridge, UK

24 University of Geneva, Geneva, Switzerland

25 Institute of Geology and Geophysics, Chinese Academy of Sciences, Beijing, China 